\title{
Imprints of an extended Chevallier-Polarski-Linder parametrization on the large scale of our universe
}

\author{
Supriya Pan ${ }^{1, \mathrm{a}}$, Weiqiang Yang ${ }^{2, \mathrm{~b}}$, Andronikos Paliathanasis ${ }^{3,4, \mathrm{c}}$ \\ ${ }^{1}$ Department of Mathematics, Presidency University, 86/1 College Street, Kolkata 700073, India \\ 2 Department of Physics, Liaoning Normal University, Dalian 116029, People's Republic of China \\ ${ }^{3}$ Institute of Systems Science, Durban University of Technology, PO Box 1334, Durban 4000, Republic of South Africa \\ ${ }^{4}$ Instituto de Ciencias Fiisicas y Matemáticas, Universidad Austral de Chile, Valdivia 5090000, Chile
}

Received: 22 August 2019 / Accepted: 14 March 2020 / Published online: 26 March 2020

(C) The Author(s) 2020

\begin{abstract}
In the present work we perform a systematic analysis of a new dark energy parametrization and its various corrections at first and higher orders around the presence epoch $z=0$, where the first order correction of this dark energy parametrization recovers the known ChevallierPolarski-Linder model. We have considered up to the third order corrections of this parametrization and investigate the models at the level of background and perturbations. The models have been constrained using the latest astronomical datasets from a series of potential astronomical data, such as the cosmic microwave background observations, baryon acoustic oscillations measurements, recent Pantheon sample of the supernova type Ia and the Hubble parameter measurements. From the analyses we found that all parametrization favor the quintessential character of the dark energy equation of state where the phantom crossing is marginally allowed (within 68\% CL). Finally, we perform the Bayesian analysis using MCEvidence to quantify the statistical deviations of the parametrizations compared to the standard $\Lambda \mathrm{CDM}$ cosmology. The Bayesian analysis reports that $\Lambda \mathrm{CDM}$ is favored over all the DE parametrizations.
\end{abstract}

\section{Introduction}

According to the theory of general relativity, one possible way to describe the recent observational evidences is to introduce the dark energy, a hypothetical fluid with large negative pressure [1]. However, apart from this negativity condition on the pressure of dark energy, no one knows what exactly this particular fluid is. The simplest explanation to the dark energy

\footnotetext{
a e-mail: supriya.maths@presiuniv.ac.in

be-mail: d11102004@163.com

ce-mail: anpaliat@phys.uoa.gr (corresponding author)
}

theory comes through the introduction of positive cosmological constant, $\Lambda$, which does not evolve with the time. But, the cosmological constant already suffers from two major problems, one which is recognized as the cosmological constant problem and the other is the cosmic coincidence problem. Thus, although as stated by a series of observational data, the $\Lambda$-cosmology is an elegant version to model the recent observational features of the universe, the problems associated with the above motivate us to think of the scenarios beyond the standard $\Lambda$-cosmology paradigm.

The simplest extension to $\Lambda$-cosmology is the $w_{x}$ cosmology in which $w_{x}$ is the dark energy equation-of-state quantified as the ratio of pressure to its density, mathematically which is $w_{x}=p_{x} / \rho_{x}$. One can identify that $p_{x}$ and $\rho_{x}$ are respectively the pressure and energy density of the dark energy fluid. The equation-of-state $w_{x}$ being -1 recovers the $\Lambda$-cosmology. In general one can assume $w_{x}(\neq-1)$ to be either time independent or dependent while the latter scenario is the most general one. Thus, in the present work we shall focus on the alternative cosmologies to the $\Lambda$-cosmology in which the dark energy equation-of-state is evolving with the expansion of the universe.

The parametrization of $w_{x}$ could be any function of the redshift $z$ or the scale factor $a(t)$ of the Friedmann-LemaitreRobertson-Walker universe; note that, $1+z=a_{0} / a(t)$, where $a_{0}$ is the present value of the scale factor in this universe. Thus, since $w_{x} \equiv w_{x}(z) \equiv w_{x}(a)$ could be any arbitrary function of the redshift or the scale factor, therefore, in principle this gives us a complete freedom to pick up any particular model of interest and test it with the observational data in order to see whether that model is able to correctly describe the evolution of the universe. In fact one can realize that the introduction of the dark energy equation-of-state is a reverse mechanism to probe the expansion history of the universe. Going back to literature, one can find that this par- 
ticular area of cosmology has been investigated well both at the level of background and perturbations where various parametrizations for $w_{x}$ were introduced earlier [2-12] and later [13-42]. Precisely, the dark energy parametrization with only a single free parameter, with two free parameters, with three free parameters and finally with more than three parameters have been rigorously studied by various investigators.

The aim of the present work is slightly different. Here, we are considering an exponential dark energy parametrization that in its first order approximation around $z=0$ recovers the CPL parametrization, and further we allow its higher order corrections in order to investigate how such extended corrections affect the evolution of the universe both at the level of background and perturbations. More specifically, we consider upto the third order expansion of the exponential dark energy model. We remark that in general every analytic function for the equation-of-state parameter around the $z=0$ describes the CPL parametrization in the first correction; however, while we want to assume a general Taylor expansion of an analytic function $f(a)$ around $a=1$, i.e. $f(a)=\sum_{i=0}^{\infty} w_{i}(a-1)^{i}$, every new term which is introduced in the correction provides a new degree of freedom, a free parameter, in the model. Consequently, the models will have different degrees of freedom and they will not be in comparison. Hence, special relations amount the constants $w_{i}$ should be considered, and for our analysis we assume that $w_{0}$ is free while $w_{j}=\frac{w_{1}}{j !}$, which $j \neq 0$, in which $f(a)$ is now the exponential function. However, by this approach we will get a remarkable information on how the nonlinear terms in the parametrizations of the equation-of-state affect the viability of the model in higher-redshifts.

The work has been organized in the following way. In Sect. 2 we introduce the models for $w_{x}(z)$ and describe the general equations at the level of background and perturbations. After that in Sect. 3 we provide an equivalence of the present dark energy parametrizations with the scalar field theory. Then in Sect. 4 we describe the observational data and the statistical analysis that are used to constrain the models. After that in Sect. 5 we describe the observational constraints extracted from the models using the astronomical data described in Sect. 4. Then in Sect. 6 we compute the evidences of the dark energy parametrizations through the MCEvidence. Finally, we close the work in Sect. 7 with a brief summary of everything.

\section{Basic equations and the models}

Considering a spatially flat Friedmann-Lemaître-RobertsonWalker line element $d s^{2}=-d t^{2}+a^{2}(t) \sum_{i=1}^{3} d x_{i}^{2}$ (where $a(t)$ is the expansion scale factor of the universe), in the context of the Einstein gravity, we assume that (i) matter is minimally coupled to gravity, (ii) there is no interaction between any two fluids under consideration and (iii) all the fluids satisfy barotropic equation of state, i.e., $p_{i}=w_{i} \rho_{i}$ in which $w_{i}$ being the barotropic state parameter for the $i$-th fluid having $\left(\rho_{i}, p_{i}\right)$ as its the energy density and pressure, respectively. Precisely, we consider that the total energy density of the universe is, $\rho_{\text {tot }}=\rho_{r}+\rho_{b}+\rho_{c}+\rho_{x}$ and the total pressure thus becomes $p_{\text {tot }}=p_{r}+p_{b}+p_{c}+p_{x}$. Here, the subscripts $r, b, c$ and $x$ respectively stands for radiation, baryons, cold dark matter and dark energy. Thus, the barotropic indices are, $w_{r}=1 / 3, w_{b}=w_{c}=0$ and we assume $w_{x}$ to be dynamical. The Einstein's field equations for the above FLRW universe can be written down as

$$
\begin{aligned}
H^{2} & =\frac{8 \pi G}{3} \rho_{t o t}, \\
\dot{H} & =-4 \pi G\left(p_{t o t}+\rho_{t o t}\right),
\end{aligned}
$$

in which an overhead dot represents the cosmic time differentiation and $H \equiv \dot{a} / a$ is the Hubble rate of this universe. Now, using (1) and (1) (or alternatively the Bianchi's identity), one can find the balance equation

$\dot{\rho}_{t o t}+3 H\left(p_{t o t}+\rho_{t o t}\right)=0$.

Now, since as we assumed that we don't have any interaction between any two fluids of the universe, thus, they should satisfy their own conservation equation leading to

$\dot{\rho}_{i}+3 H\left(p_{i}+\rho_{i}\right)=0$,

from which using the relation between pressure and energy density for the radiation, baryons, and cold (pressureless-) dark matter, one can find that $\rho_{r}=\rho_{r 0} a^{-4}, \rho_{m}=\rho_{b}+\rho_{c}=$ $\left(\rho_{b 0}+\rho_{c 0}\right) a^{-3}$. Here, $\rho_{i 0}$ is the present value of $\rho_{i}$. And finally, the evolution of the dark energy fluid can be given by,

$\rho_{x}=\rho_{x 0}\left(\frac{a}{a_{0}}\right)^{-3} \exp \left(-3 \int_{a_{0}}^{a} \frac{w_{x}\left(a^{\prime}\right)}{a^{\prime}} d a^{\prime}\right)$,

where $\rho_{x 0}$ being the current value of $\rho_{x}$ and $a_{0}$ is the present value of the scale factor that we set to be unity $\left(a_{0}=1\right)$ without any loss of generality. We further note that the scale factor is related to the redshift that we shall frequently use hereafter via $1+z=a_{0} / a=1 / a$. Thus, once the dark energy equation of state is prescribed, the evolution of the dark energy density can be found.

As we discussed above, we consider that the dark energy fluid follows a general parametrization in the following way:

$w_{x}(z)=\left(w_{0}-w_{a}\right)+w_{a} \exp \left(\frac{z}{1+z}\right)$

where $w_{0}$ is the present value of the dark energy equation of state, that means, $w_{x}(z=0)=w_{0}$ and $w_{a}$ is another free parameter. The model (6) is very interesting by its construction since one can easily recognize that it could return a number of interesting parametrization that 
includes the classic Chevallier-Polarski-Linder parametrization $w_{x}(z)=w_{0}+w_{a} z /(1+z)$ if we take the first approximation of the exponential function in (6).

We expand the exponential function of (6) upto its first, second and third order corrections leading to the following class of dark energy parametrization:

$$
\begin{aligned}
& w_{x}(a)=w_{0}+w_{a} \frac{z}{1+z} \\
& w_{x}(a)=w_{0}+w_{a}\left[\frac{z}{1+z}+\frac{1}{2 !}\left(\frac{z}{1+z}\right)^{2}\right] \\
& w_{x}(a)=w_{0}+w_{a}\left[\frac{z}{1+z}+\frac{1}{2 !}\left(\frac{z}{1+z}\right)^{2}+\frac{1}{3 !}\left(\frac{z}{1+z}\right)^{3}\right]
\end{aligned}
$$

and for convenience we call the dark energy parametrization of Eqs. (7), (8) and (9) as "Extension 1" (Ext1 in short), "Extension 2" (Ext2 in short) and "Extension 3" (Ext3 in short), respectively. Let us note that in the above cases we have not considered the pivot redshift [39]. However, the consideration of pivoting redshift might be an interesting issue for investigations because as already commented in [39], one can find a specific value of the pivot redshift where the parameters $w_{0}$ and $w_{a}$ are uncorrelated.

At the end of this section, we would like to present the qualitative features of the present dark energy parametrizations in terms of the evolution of their equations of state and the deceleration parameters. In order to do so, we assumed three different values of $w_{0}$, namely, $w_{0}=-0.95, w_{0}=-1$ and $w_{0}=-1.1$ and in each case we consider various values of $w_{a}$ to understand how the curves behave with the increasing of the $w_{a}$ parameter. In Fig. 1 we show the evolution of the dark energy parameterizations (6), (7), (8) and (9) set- ting the present value of the dark energy equation of state at $w_{0}=0.95$ where we allow different values of $w_{a}$ such as $w_{a}=-0.3,-0.2,-0.1,0,0.1,0.2,0.3$. The curve with $w_{a}=0$ simply returns $w=w_{0}$ and this has been kept to compare with other curves having $w_{a} \neq 0$. From Fig. 1, we notice that for $w_{a}<0$, the dark energy equation of state allows its phantom character which is much pronounced at high redshifts, while for $w_{a}>0$, the reverse scenario is found. In a similar fashion, we investigated the other cases with $w_{0}=-1$ and $w_{0}=-1.1$, however, we did not observe any significant changes in the qualitative evolution of $w_{x}(z)$, so we did not include the other figures.

We then plot the evolution of the deceleration parameter for all the DE parametrizations, namely, (6), (7), (8) and (9). Here we again assumed three fixed values of $w_{0}$, namely, $w_{0}=-0.95,-1,-1.1$ and in each case we assume different values of $w_{a}$ similar to what we have shown in Fig. 1. Finally, we analyzed the evolution of the deceleration parameter for all the cases and found that all three cases return almost similar dynamics. That is why choose the case with for $w_{0}=-0.95$ and exclude the others. The Fig. 2 corresponds to the case $w_{0}=-0.95$. From this figure one can see that irrespective of the values of $w_{0}$, a fine transition from the past decelerating phase to the current accelerating one is observed. The interesting and important point in Fig. 2 is that, for negative values of $w_{a}$ the transition redshifts are shifting towards higher redshifts (although mild) while for positive values of $w_{a}$, we see the reverse, that means the transition redshifts are shifting towards lower values of the redshift.

Overall, we find that the models at the level of background do not exhibit any deviations from one another. This is not surprising because the deviations between the cosmological models are usually reflected from their analysis at the level
Fig. 1 We show the evolution of the dark energy parametrizations for different values of $w_{a}$ with a fixed value of $w_{0}=-0.95$
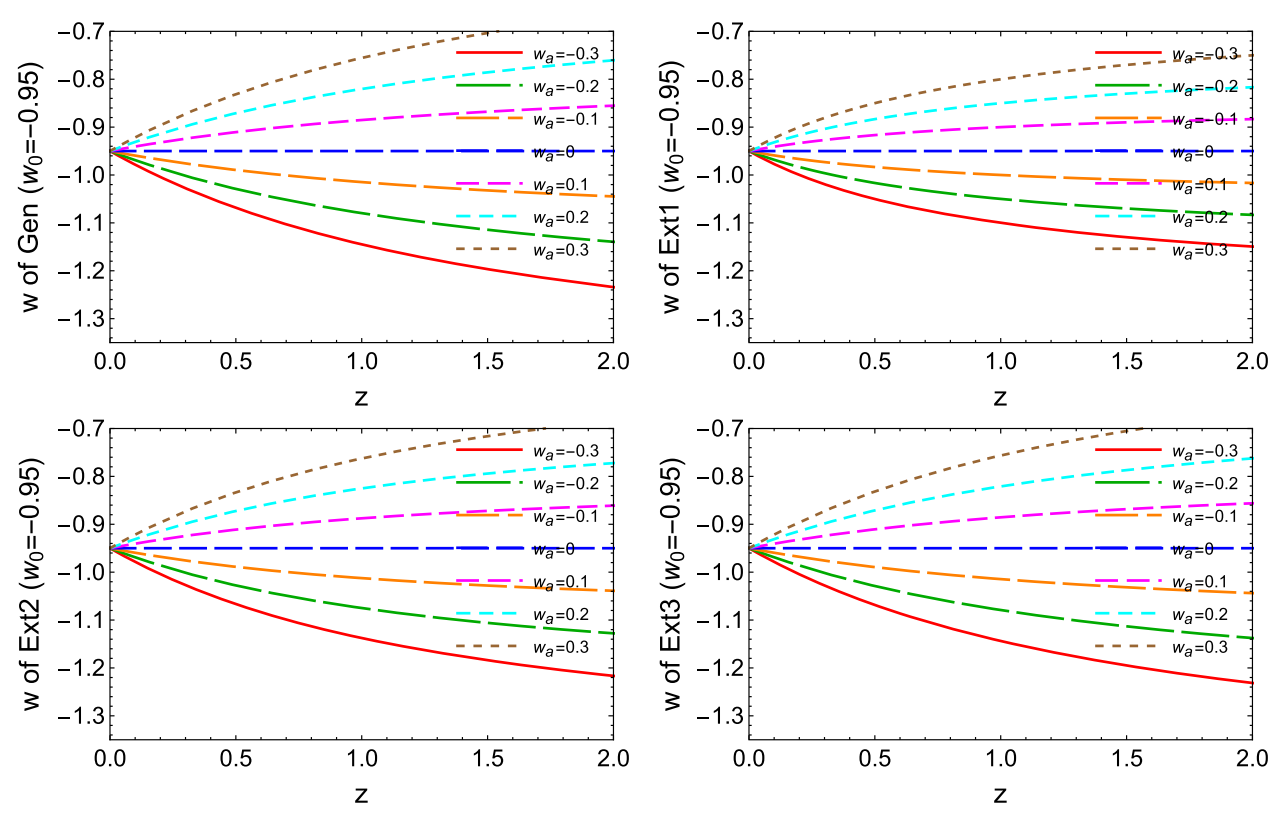
Fig. 2 The evolution of the deceleration parameter depicting a clear transition from the past decelerating phase to the current accelerating phase for all the dark energy models has been presented for different values of $w_{a}$ and with a fixed value of $w_{0}=-0.95$. One can easily notice that as long as $w_{a}$ increase from its negative to positive values, the transition redshift shifts more closer to the present epoch
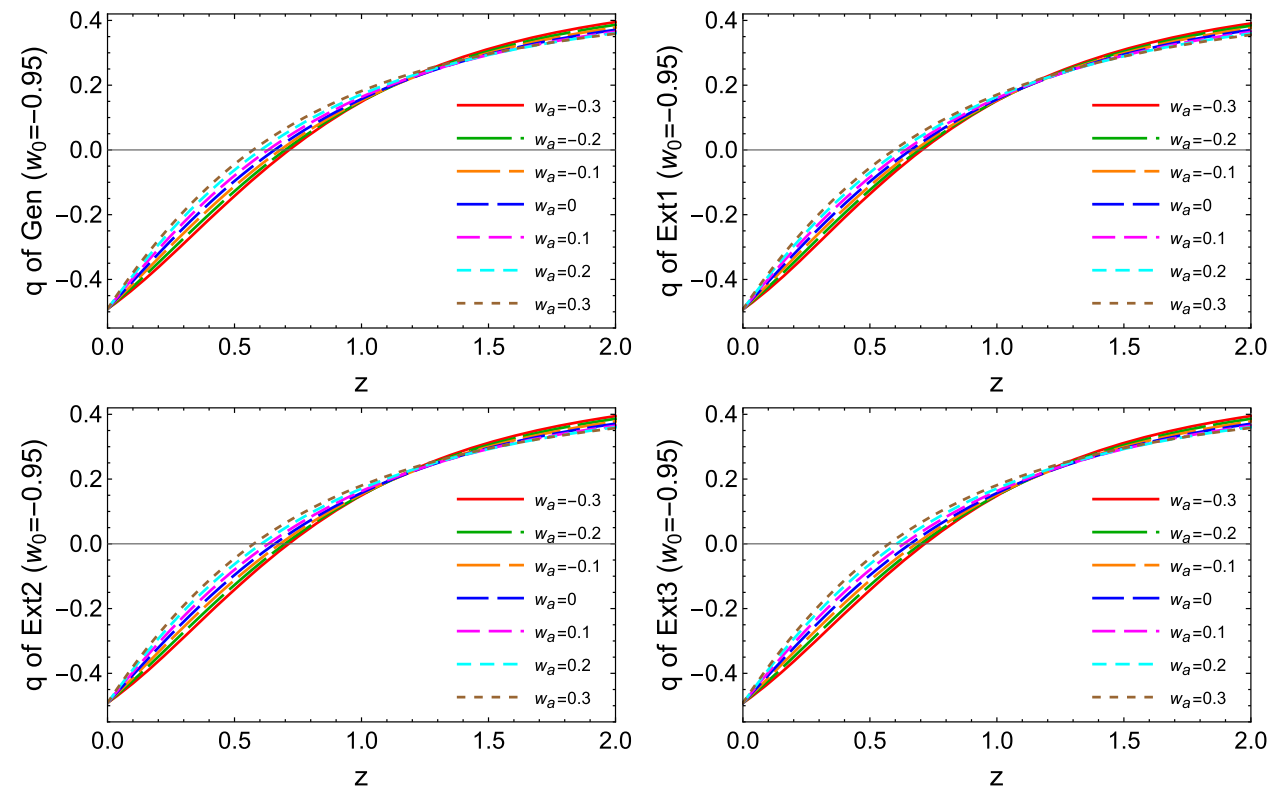

of perturbations. In what follows we shall consider the perturbation equations for all the DE parametrizations in this work.

We start with the following metric which is the perturbed form of the FLRW line element:

$d s^{2}=a^{2}(\eta)\left[-d \eta^{2}+\left(\delta_{i j}+h_{i j}\right) d x^{i} d x^{j}\right]$

Here, $\eta$ denotes the conformal time; $\delta_{i j}, h_{i j}$ are the unperturbed and the perturbative metric tensors, respectively. Now, considering the perturbed Einstein's field equations, for a mode with wave-number $k$ one can write down [43-45]:

$$
\begin{aligned}
\delta_{i}^{\prime}= & -\left(1+w_{i}\right)\left(\theta_{i}+\frac{h^{\prime}}{2}\right)-3 \mathcal{H}\left(\frac{\delta p_{i}}{\delta \rho_{i}}-w_{i}\right) \delta_{i} \\
& -9 \mathcal{H}^{2}\left(\frac{\delta p_{i}}{\delta \rho_{i}}-c_{a, i}^{2}\right)\left(1+w_{i}\right) \frac{\theta_{i}}{k^{2}}, \\
\theta_{i}^{\prime}= & -\mathcal{H}\left(1-3 \frac{\delta p_{i}}{\delta \rho_{i}}\right) \theta_{i}+\frac{\delta p_{i} / \delta \rho_{i}}{1+w_{i}} k^{2} \delta_{i}-k^{2} \sigma_{i},
\end{aligned}
$$

where $\delta_{i}=\delta \rho_{i} / \rho_{i}$ is the density perturbation for the $i$-th fluid; the prime associated to any quantity denotes the derivatives with respect to conformal time; $\mathcal{H}=a^{\prime} / a$ is the conformal Hubble parameter; $\theta_{i} \equiv i k^{j} v_{j}$ is the divergence of the $i$-th fluid velocity; $h=h_{j}^{j}$, is the trace of the metric perturbations $h_{i j} ; \sigma_{i}$ denotes the anisotropic stress related to the $i$-th fluid. Let us also note that $c_{a, i}^{2}=\dot{p}_{i} / \dot{\rho}_{i}$, is the adiabatic speed of sound of the $i$-th fluid which can also be written in terms of other physical quantities as $c_{a, i}^{2}=w_{i}-\frac{w_{i}^{\prime}}{3 \mathcal{H}\left(1+w_{i}\right)}$, where we fix the sound speed $c_{s}^{2}=\delta p_{i} / \delta \rho_{i}$ to be unity. Finally, we also note that we have neglected the anisotropic stress from the system for simplicity.

\section{Scalar-field description}

This section is devoted to provide with an equivalent field theoretic description for the dark energy parametrizations. A method to construct the scalar field potential which describe a given equation of state parameter was presented in [46]. Specifically, for a spatially flat FLRW as in the case of consideration, with a line element

$$
\begin{aligned}
d s^{2}= & -e^{F(\omega)} d \omega^{2}+e^{\omega / 3}\left[\frac{d r^{2}}{1-K r^{2}}+r^{2} d \theta^{2}\right. \\
& \left.+r^{2} \sin ^{2} \theta d \phi^{2}\right]
\end{aligned}
$$

where $e^{F(\omega)}$ plays the role of a lapse function, the scale factor $a(\omega)=e^{\omega / 3}$ and $K$ is the curvature scalar of the universe. The exact solution of the scalar field and the scalar field potential in case of vacuum are

$$
\begin{gathered}
\phi(\omega)= \pm \frac{\sqrt{6}}{6} \int \sqrt{F^{\prime}(\omega)} d \omega, \\
V(\omega)=\frac{1}{12} e^{-F(\omega)}\left(1-F^{\prime}(\omega)\right)
\end{gathered}
$$

or, equivalently, for the physical parameters, such as energy density and pressure

$\rho_{\phi}(\omega)=\frac{1}{12} e^{-F(\omega)}, P_{\phi}(\omega)=\frac{1}{12} e^{-F(\omega)}\left(2 F^{\prime}(\omega)-1\right)$.

Consequently, for the latter definitions it follows

$w_{\phi}(\omega)=\frac{P_{\phi}(\omega)}{\rho_{\phi}(\omega)}=\left(2 F^{\prime}(\omega)-1\right)$.

Hence, for a specific equation of state parameter $w_{\phi}(\omega)$ the latter first-order equation can be solved and we can deter- 
mine the function $F(\omega)$. Subsequently, by replacing $F(\omega)$ in Eqs. (13), (14) one can find the functional form of $V(\phi)$. For the general functional form of the equation of state, namely, Eq. (6), $w_{x}(\omega)=w_{0}-w_{a}+w_{a} \exp \left(1-e^{\omega / 3}\right)$, we find that

$$
\begin{aligned}
F(\omega)= & \frac{1}{2}\left(1+w_{0}-w_{a}\right) \omega \\
& +\frac{1}{2} w_{a} \int \exp \left(1-e^{\omega / 3}\right) d \omega+F_{0},
\end{aligned}
$$

where $F_{0}$ is the constant of integration. Now, using the value of $F(\omega)$, one can find that

$$
\phi(\omega)=\frac{1}{\sqrt{2}} \int \sqrt{1+w_{0}-w_{a}+w_{a} \exp \left(1-e^{\omega / 3}\right)} d \omega,
$$

and $V(\omega)$ can be solved as

$$
\begin{aligned}
V(\omega)= & \frac{1}{24}\left(-e^{e^{\omega / 3}}\left(w_{0}+w_{a}-1\right)-e w_{a}\right) \\
& \times \exp \left(-\frac{3 e}{2} w_{a} \operatorname{Ei}\left(-e^{\omega / 3}\right)\right. \\
& \left.-\frac{\omega}{2}\left(w_{0}+w_{a}+1\right)-e^{\omega / 3}\right),
\end{aligned}
$$

where the symbol 'Ei' represents the exponential integral. For the CPL potential $w_{x}(\omega)=w_{0}+w_{a}\left(1-e^{\omega / 3}\right)$ we find

$F(\omega)=\frac{1}{2}\left(1+w_{0}+w_{a}\right) \omega-\frac{3}{2} w_{a} e^{\omega / 3}+F_{0}$,

where $F_{0}$ is the integration constant and consequently we find that

$$
\begin{aligned}
\frac{\phi(\omega)}{\sqrt{3}}= & \sqrt{w_{a}\left(e^{w / 3}-1\right)-\left(1+w_{0}\right)}-\sqrt{1+w_{0}+w_{a}} \\
& \times \arctan \sqrt{\frac{w_{a}\left(e^{w / 3}-1\right)-\left(1+w_{0}\right)}{1+w_{0}+w_{a}}}
\end{aligned}
$$

$$
\begin{aligned}
V(\omega)= & \frac{1}{24} e^{F_{0}} \exp \left(\frac{3}{2} w_{a} e^{\omega / 3}-\frac{1}{2}\left(1+w_{0}+w_{a}\right)\right) \\
& \times\left(1-w_{0}+w_{a}\left(e^{\omega / 3}-1\right)\right) .
\end{aligned}
$$

For the Ext 2 and Ext 3 models the corresponding functions $F(\omega)$ are derived to be

$$
\begin{aligned}
F_{E x t}^{2}(\omega)= & F_{0}+\frac{1}{4}\left(\left(2+2 w_{0}+3 w_{a}\right) \omega\right. \\
& \left.-12 w_{a} e^{\omega / 3}+\frac{3}{2} w_{a} e^{2 \omega / 3}\right), \\
F_{E x t}^{3}(\omega)= & F_{0}+\frac{1}{12}\left(\left(6 \omega+6 w_{0}+10 w_{a}\right) \omega\right. \\
& \left.-w_{a}\left(e^{\omega}+45 e^{\omega / 3}-9 e^{2 \omega / 3}\right)\right) .
\end{aligned}
$$

In Figs. 3, 4 and 5 the qualitative evolution of the scalar field equivalent $\phi(\omega)$, the scalar field potential $V(\omega)$ and the parametric plot $V(\omega)$ are presented respectively for the CPL parametrization (Ext1) and the other two extensions, namely, Ext2, and Ext3.

\section{Observational data}

For the convenience of the reader and for our presentation we provide the details of the observational data used to constrain the dynamical dark energy parametrization and also the methodology.

- Cosmic microwave background observations: the cosmic microwave background (CMB) observations are one of the powerful data to probe the nature of dark energy. Here we use the CMB from Planck 2015 [47,48]. The high$\ell$ temperature and polarization data as well as the low$\ell$ temperature and polarization data from Planck 2015
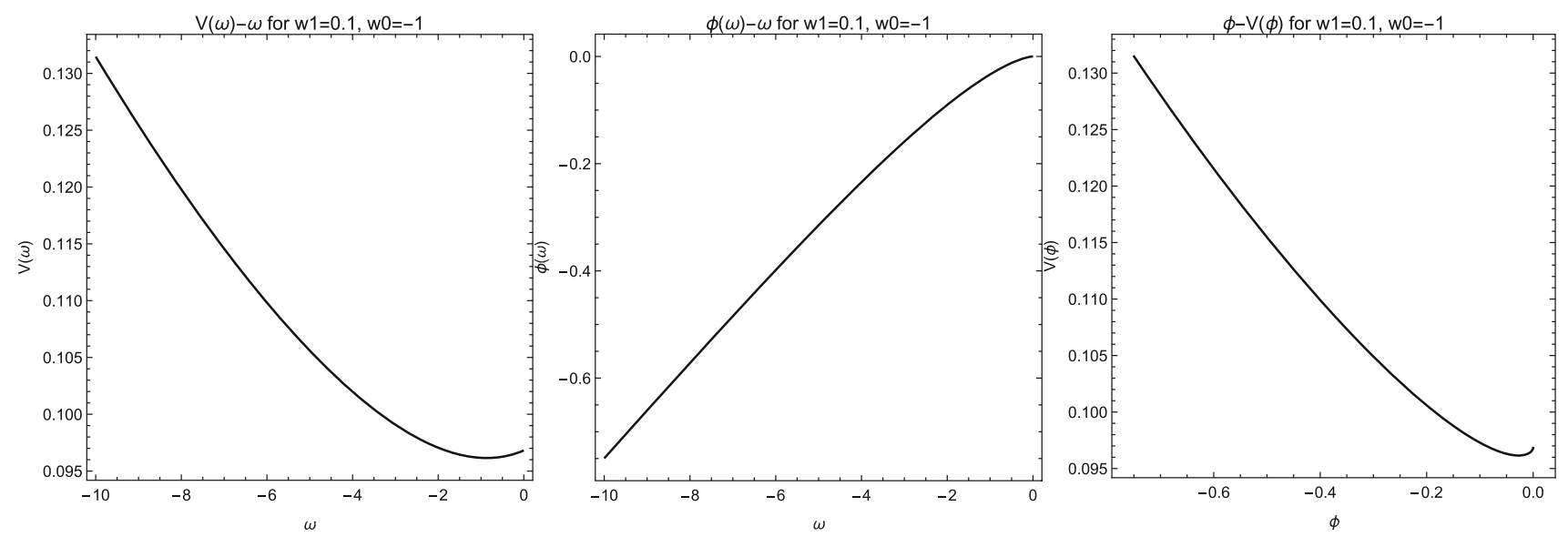

Fig. 3 Qualitative evolution of the scalar field $\phi(\omega)$, the scalar field potential $V(\omega)$ as also the parametric plot $\phi-V(\phi)$ is given for the CPL (Ext1) model 

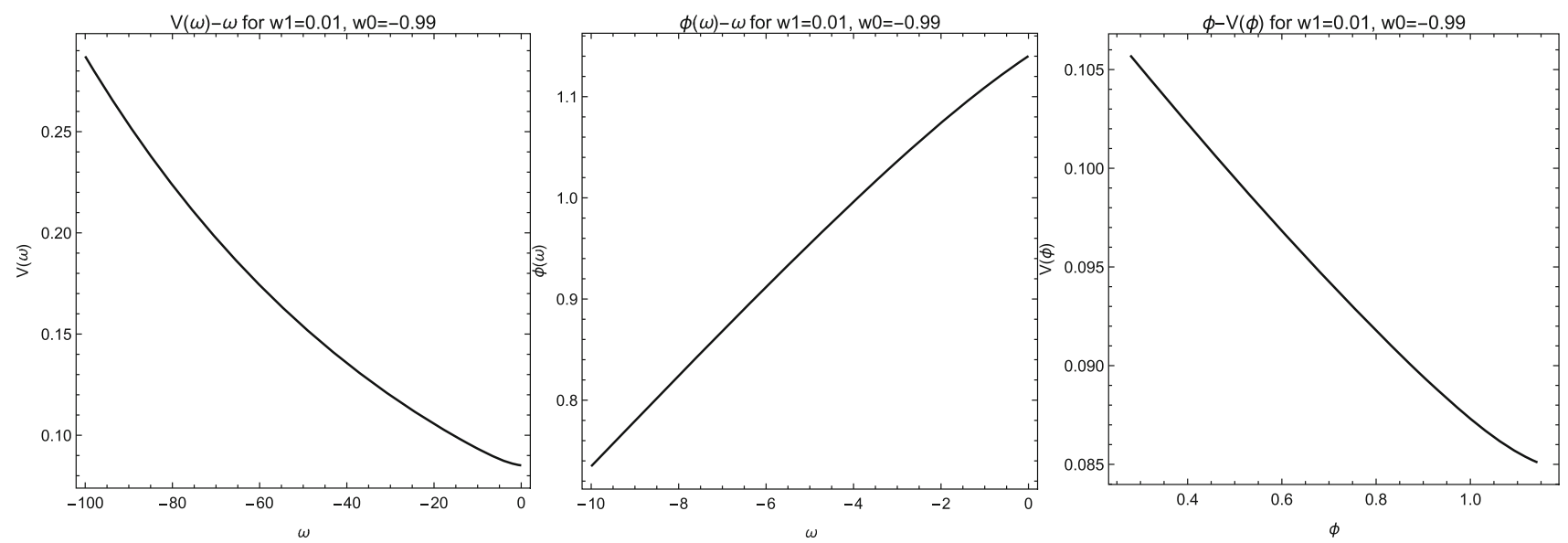

Fig. 4 Qualitative evolution of the scalar field $\phi(\omega)$, the scalar field potential $V(\omega)$ as also the parametric plot $\phi-V(\phi)$ is given for the Ext2 model
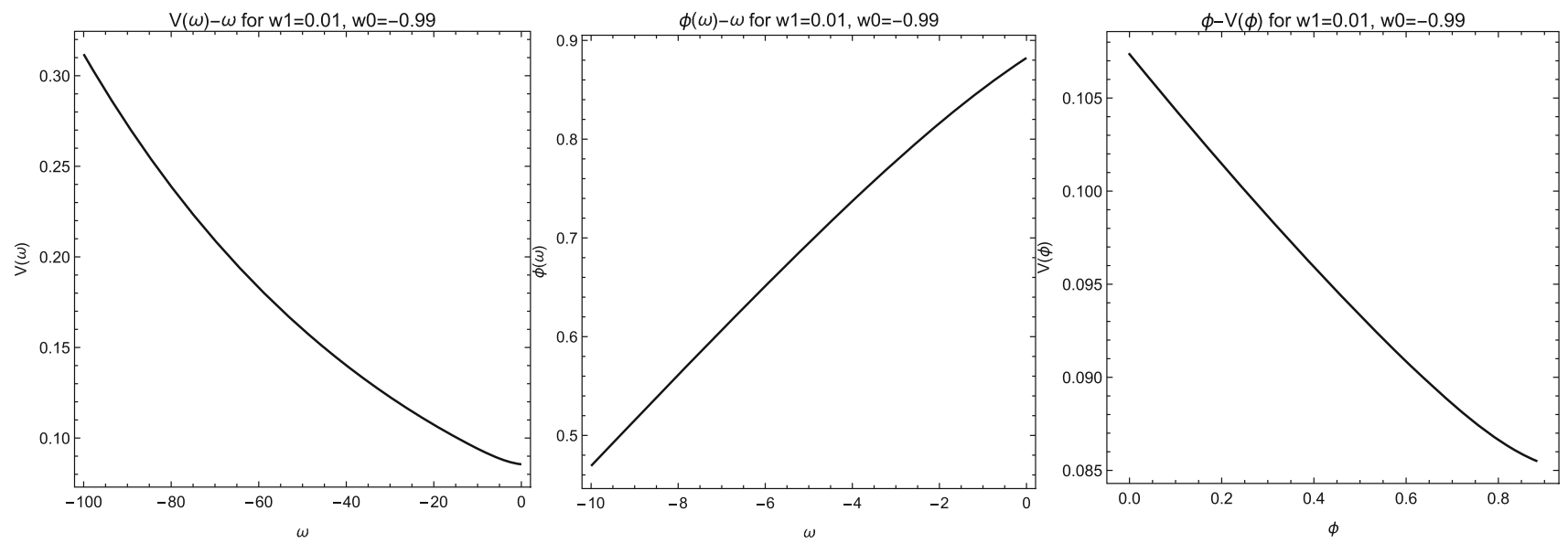

Fig. 5 Qualitative evolution of the scalar field $\phi(\omega)$, the scalar field potential $V(\omega)$ as also the parametric plot $\phi-V(\phi)$ is given for the Ext3 model

(precisely the dataset: Planck TT, TE, EE + lowTEB) $[47,48]$ have been considered.

- Baryon acoustic oscillations: The baryon acoustic oscillations (BAO) data from different superovulation missions are used [50-52].

- Supernovae Type Ia: We also use latest released Pantheon sample [53] from the Supernovae Type Ia.

- Hubble parameter measurements: Finally, we use the Hubble parameter measurements from the Cosmic Chronometers (CC) [54].

Now we come to the technical part of the statistical analysis. Thus, we have performed the fitting analysis using the modified version of cosmomc [55,56], an efficient Markov chain monte carlo package equipped with a convergence diagnostic given by the Gelman and Rubin statistics [57]. This package includes the support for the Planck 2015 likelihood code [48] (see http://cosmologist.info/cosmomc/). In Table 1 we have shown the flat priors on the model param-
Table 1 The table shows the flat priors on the model parameters used during the statistical analysis

\begin{tabular}{ll}
\hline Parameter & Prior \\
\hline$\Omega_{b} h^{2}$ & {$[0.005,0.1]$} \\
$\Omega_{c} h^{2}$ & {$[0.01,0.99]$} \\
$\tau$ & {$[0.01,0.8]$} \\
$n_{s}$ & {$[0.5,1.5]$} \\
$\log \left[10^{10} A_{s}\right]$ & {$[2.4,4]$} \\
$100 \theta_{M C}$ & {$[0.5,10]$} \\
$w_{0}$ & {$[-2,0]$} \\
$w_{a}$ & {$[-3,3]$} \\
\hline
\end{tabular}

eters that have been used during the observational analysis. Perhaps it might be important to mention here that in the present analysis we have used Planck 2015 likelihood [48] instead of Planck 2018 likelihood (although the cosmological parameters from Planck 2018 are already available [58]) because Planck 2018 likelihood code is not public yet. However, it will be worth to run the same codes that we use for 
Table 2 Observational constraints on various free parameters at $68 \%$ and $95 \% \mathrm{CL}$ for the dynamical dark energy state parameter $w_{x}(z)=$ $\left(w_{0}-w_{a}\right)+w_{a} \exp \left(\frac{z}{1+z}\right)$ (Gen) using different astronomical datasets have been presented. Let us note that $\Omega_{m 0}$ is the present value of $\Omega_{m}=\Omega_{c}+\Omega_{b}$ and $H_{0}$ is in the units of $\mathrm{km} / \mathrm{sec} / \mathrm{Mpc}$

\begin{tabular}{|c|c|c|c|}
\hline Parameters & $\mathrm{CMB}+\mathrm{BAO}$ & $\mathrm{CMB}+\mathrm{BAO}+$ Pantheon & $\mathrm{CMB}+\mathrm{BAO}+$ Pantheon+CC \\
\hline$\Omega_{c} h^{2}$ & $0.1194_{-0.0014-0.0026}^{+0.0013+0.0027}$ & $0.1193_{-0.0013-0.0025}^{+0.0013+0.0024}$ & $0.1195_{-0.0013-0.0026}^{+0.0013+0.0026}$ \\
\hline$\Omega_{b} h^{2}$ & $0.02222_{-0.00016-0.00030}^{+0.00015+0.00029}$ & $0.02223_{-0.00015-0.00029}^{+0.00015+0.00029}$ & $0.02222_{-0.00015-0.00030}^{+0.00014+0.00030}$ \\
\hline $100 \theta_{M C}$ & $1.04049_{-0.00032-0.00066}^{+0.00032+0.00063}$ & $1.04051_{-0.00031-0.00062}^{+0.00030+0.00063}$ & $1.04049_{-0.00032-0.00063}^{+0.00032+0.00066}$ \\
\hline$\tau$ & $0.079_{-0.018-0.035}^{+0.018+0.033}$ & $0.080_{-0.017-0.034}^{+0.018+0.034}$ & $0.079_{-0.018-0.034}^{+0.018+0.034}$ \\
\hline$n_{s}$ & $0.9738_{-0.0044-0.0085}^{+0.0044+0.0086}$ & $0.9740_{-0.0041-0.0082}^{+0.0041+0.0083}$ & $0.9735_{-0.0042-0.0078}^{+0.0042+0.0080}$ \\
\hline $\ln \left(10^{10} A_{s}\right)$ & $3.100_{-0.034-0.068}^{+0.035+0.064}$ & $3.103_{-0.034-0.067}^{+0.034+0.066}$ & $3.101_{-0.035-0.066}^{+0.034+0.066}$ \\
\hline$w_{0}$ & $-0.537_{-0.441-0.526}^{+0.442+0.532}$ & $-0.963_{-0.082-0.127}^{+0.060+0.158}$ & $-0.933_{-0.070-0.135}^{+0.071+0.140}$ \\
\hline$w_{a}$ & $-1.154_{-0.807-1.298}^{+0.894+1.340}$ & $-0.231_{-0.143-0.499}^{+0.291+0.416}$ & $-0.337_{-0.204-0.493}^{+0.288+0.431}$ \\
\hline$\Omega_{m 0}$ & $0.348_{-0.035-0.056}^{+0.034+0.055}$ & $0.306_{-0.007-0.014}^{+0.007+0.015}$ & $0.306_{-0.008-0.015}^{+0.008+0.015}$ \\
\hline$\sigma_{8}$ & $0.804_{-0.033-0.050}^{+0.026+0.056}$ & $0.839_{-0.016-0.032}^{+0.018+0.031}$ & $0.840_{-0.017-0.032}^{+0.016+0.033}$ \\
\hline$H_{0}$ & $64.14_{-3.80-5.12}^{+2.51+5.60}$ & $68.24_{-0.80-1.51}^{+0.78+1.56}$ & $68.23_{-0.83-1.62}^{+0.84+1.67}$ \\
\hline
\end{tabular}

the present models but with the new Planck 2018 likelihood which will enable us to understand any effective changes in the cosmological parameters and consequently more stringent constraints on them as well.

\section{Observational constraints and the analysis}

In this section we describe the observational constraints on all the dark energy parametrization, namely the general parametrization of Eq. (6), Extension 1 or the CPL
Fig. $6 \quad 68 \%$ and $95 \% \mathrm{CL}$ contour plots for various combinations of the model parameters of the general parametrization of (6) (Gen) have been shown for different observational combinations. The figure also contains the one dimensional marginalized posterior distributions for the parameters shown in the two dimensional contour plots

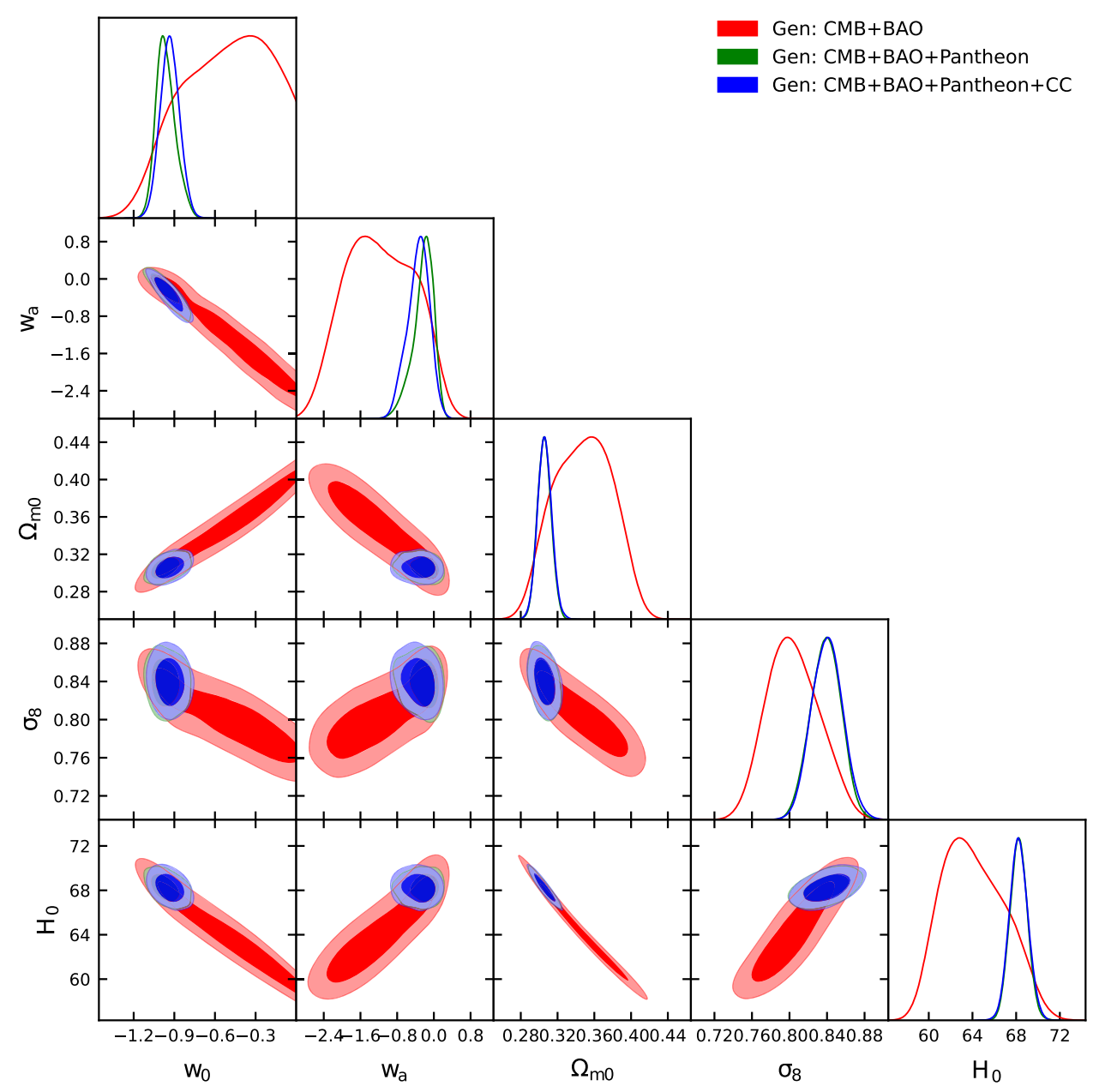


parametrization of Eq. (7), Extension 2 of Eq. (8) and extension 3 of Eq. (9) using various astronomical datasets summarized in Sect. 4. In particular, we focus on the two key parameters of the dark energy parametrization, namely, $w_{0}$ and $w_{a}$ in order to investigate the qualitative changes in the parametrization as long as nonlinear terms are considered. In what follows we describe the observational constraints extracted from each dark energy scenario.

Let us first focus on the general dark energy parametrization given in Eq. (6). We have constrained this dark energy scenario using different cosmological datasets such as $\mathrm{CMB}+\mathrm{BAO}, \mathrm{CMB}+\mathrm{BAO}+\mathrm{Pantheon}$ and $\mathrm{CMB}+\mathrm{BAO}+$ Pantheon $+\mathrm{CC}$, the results of which are summarized in Table 2. From Table 2, a general conclusion that one might quickly observe is that, the inclusion of Pantheon to $\mathrm{CMB}+\mathrm{BAO}$ significantly improves the error bars on all the parameters, and not only that, some of the parameters are significantly improved concerning their mean values. In fact, the best constraints on the model parameters are achieved for the combination $\mathrm{CMB}+\mathrm{BAO}+\mathrm{Pantheon}$. The inclusion of $\mathrm{CC}$ to $\mathrm{CMB}+\mathrm{BAO}+$ Pantheon although does not add much significant insight on the cosmological constraints, however, the effects on $w_{a}$ are pronounced when $\mathrm{CC}$ data are added to $\mathrm{CMB}+\mathrm{BAO}+$ Pantheon. Let us now focus on the constraints on individual model parameters. As one can see from Table 2 that the mean value of the dark energy equation of state at present, i.e., $w_{0}$ is always in the quintessential regime: $w_{0}=-0.963_{-0.082}^{+0.060}$ at $68 \% \mathrm{CL}$ for $\mathrm{CMB}+\mathrm{BAO}+\mathrm{Pantheon}$ and $w_{0}=-0.933_{-0.070}^{+0.071}$ at $68 \%$ $\mathrm{CL}$ for $\mathrm{CMB}+\mathrm{BAO}+$ Pantheon+CC. Although from statistical point of view, one can argue that the constraints on $w_{0}$ mildly suggest for a crossing of the phantom divide line $w_{0}=-1$, however, $w_{0}=-1$ is the most consistent scenario. Concerning the remaining key parameter, $w_{a}$, we find that it may assume non null values, however, $w_{a}=0$ is allowed within $68 \% \mathrm{CL}$ of course. In Fig. 6, we have shown the one dimen- sional posterior distributions for some selected parameters of this model as well as the two dimensional contour plots considering various combinations of the model parameters. From Fig. 6, one can see that the parameters shown in this figure are correlated with each other. Specifically, we find a strong correlation between $w_{0}, w_{a}$ and $H_{0}$. Finally, we focus on the estimation of the Hubble constant $H_{0}$ for all three datasets. One can strikingly see that for $\mathrm{CMB}+\mathrm{BAO}, H_{0}$ assumes a very lower value $\left(H_{0}=64.14_{-3.80}^{+2.51}\right.$ at $\left.68 \% \mathrm{CL}, \mathrm{CMB}+\mathrm{BAO}\right)$ compared to the $\Lambda \mathrm{CDM}$ based Planck's estimation [59] and this naturally increases the tension with the local measurements [60]. However, for the remaining datasets, we find that $H_{0}$ takes higher values with slightly higher error bars compared to the $\Lambda \mathrm{CDM}$ based Planck's estimation [59], thus, it slightly decreases the tension on it.

We now consider the first extension of the general parametrization (6) that leads to the well known CPL parametrization of (7). The cosmic scenario driven by this parametrization has been constrained using the same observational datasets applied to the general DE parametrization and the numerical results are summarized in Table 3. One can clearly see that the Hubble constant takes similar values compared to the previous scenario (see Table 2). In fact, concerning the key parameters, namely, $w_{0}$ and $w_{0}$, our conclusion remains same, that means the constraints on $w_{0}$ and $w_{a}$ are almost similar to what we have found with the general parametrization (6). So, effectively we see that the first approximation (7) of the original parametrization (6) returns similar fit to the original parametrization (6). Finally, in Fig. 7 we have shown the graphical behaviour of various model parameters containing the one dimensional marginalized posterior distributions as well as the two dimensional contour plots at $68 \%$ and $95 \%$ CL.

Then we move to the observational constraints of the next parametrization given in Eq. 8. The results for this parametrization are shown in Table 4 and Fig. 8. We do not
Table 3 Observational constraints on the dark energy parametrization, namely, the Ext1 of (7) using various observational datasets. We note that $H_{0}$ is in the units of $\mathrm{km} / \mathrm{Mpc} / \mathrm{sec}$ and $\Omega_{m 0}$ is the present value of $\Omega_{m}=\Omega_{b}+\Omega_{c}$

\begin{tabular}{|c|c|c|c|}
\hline Parameters & $\mathrm{CMB}+\mathrm{BAO}$ & $\mathrm{CMB}+\mathrm{BAO}+$ Pantheon & $\mathrm{CMB}+\mathrm{BAO}+$ Pantheon+CC \\
\hline$\Omega_{c} h^{2}$ & $0.1191_{-0.0013-0.0027}^{+0.0014+0.0026}$ & $0.1191_{-0.0013-0.0026}^{+0.0013+0.0025}$ & $0.1191_{-0.0013-0.0026}^{+0.0013+0.0026}$ \\
\hline$\Omega_{b} h^{2}$ & $0.02226_{-0.00015-0.00029}^{+0.00015+0.00029}$ & $0.02227_{-0.00015-0.00030}^{+0.00015+0.00030}$ & $0.02227_{-0.00015-0.00030}^{+0.00015+0.00030}$ \\
\hline $100 \theta_{M C}$ & $1.04078_{-0.00032-0.00064}^{+0.00033+0.00063}$ & $1.04080_{-0.00031-0.00062}^{+0.00032+0.00064}$ & $1.04080_{-0.00032-0.00062}^{+0.00031+0.00062}$ \\
\hline$\tau$ & $0.078_{-0.017-0.034}^{+0.017+0.034}$ & $0.080_{-0.017-0.035}^{+0.017+0.035}$ & $0.080_{-0.018-0.034}^{+0.018+0.034}$ \\
\hline$n_{s}$ & $0.9665_{-0.0044-0.0084}^{+0.0044+0.0091}$ & $0.9667_{-0.0044-0.0086}^{+0.0044+0.0089}$ & $0.9666_{-0.0044-0.0087}^{+0.0045+0.0087}$ \\
\hline $\ln \left(10^{10} A_{s}\right)$ & $3.090_{-0.033-0.066}^{+0.034+0.066}$ & $3.092_{-0.033-0.068}^{+0.034+0.066}$ & $3.092_{-0.034-0.066}^{+0.034+0.066}$ \\
\hline$w_{0}$ & $-0.524_{-0.236-0.514}^{+0.374+0.524}$ & $-0.947_{-0.088-0.156}^{+0.076+0.165}$ & $-0.950_{-0.084-0.152}^{+0.075+0.152}$ \\
\hline$w_{a}$ & $-1.403_{-1.021-1.466}^{+0.731+1.570}$ & $-0.308_{-0.273-0.677}^{+0.367+0.619}$ & $-0.291_{-0.256-0.588}^{+0.338+0.555}$ \\
\hline$\Omega_{m 0}$ & $0.344_{-0.026-0.054}^{+0.032+0.051}$ & $0.304_{-0.007-0.015}^{+0.008+0.016}$ & $0.304_{-0.008-0.015}^{+0.008+0.015}$ \\
\hline$\sigma_{8}$ & $0.803_{-0.030-0.051}^{+0.024+0.053}$ & $0.838_{-0.016-0.033}^{+0.018+0.032}$ & $0.837_{-0.016-0.032}^{+0.016+0.032}$ \\
\hline$H_{0}$ & $64.36_{-3.23-4.67}^{+2.05+5.26}$ & $68.34_{-0.82-1.63}^{+0.81+1.70}$ & $68.31_{-0.83-1.66}^{+0.84+1.64}$ \\
\hline
\end{tabular}




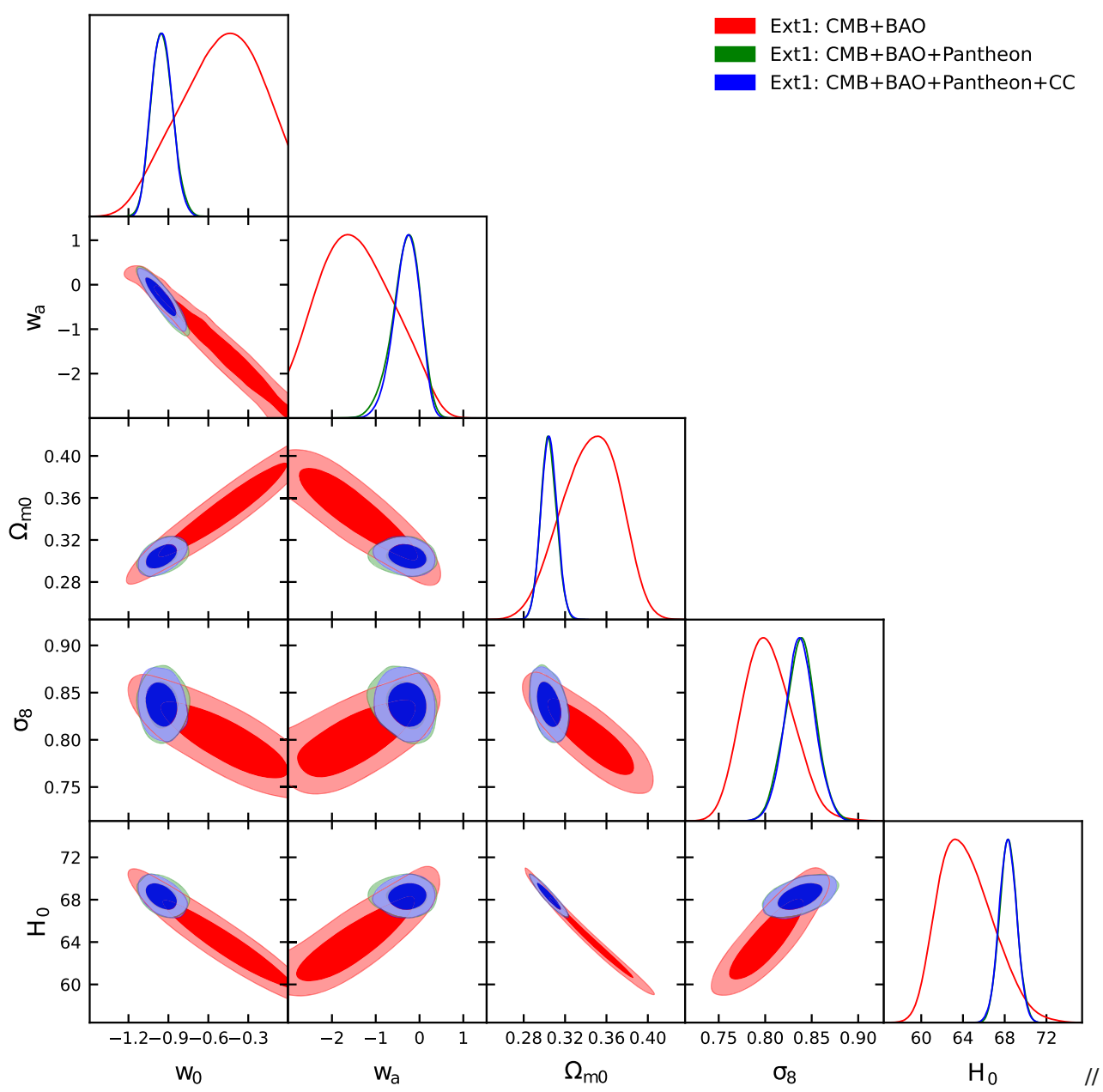

Fig. $768 \%$ and 95\% CL contour plots for various combinations of the model parameters of Ext1 of (7) [the CPL parametrization] have been shown for different observational combinations. The figure also contains the one dimensional marginalized posterior distributions for the parameters shown in the two dimensional contour plots
Table 4 Observational constraints on the dark energy parametrization, namely, the Ext 2 of (8) using various observational datasets. We note that $H_{0}$ is in the units of $\mathrm{km} / \mathrm{Mpc} / \mathrm{sec}$ and $\Omega_{m 0}$ is the present value of $\Omega_{m}=\Omega_{b}+\Omega_{c}$

\begin{tabular}{|c|c|c|c|}
\hline Parameters & $\mathrm{CMB}+\mathrm{BAO}$ & $\mathrm{CMB}+\mathrm{BAO}+$ Pantheon & $\mathrm{CMB}+\mathrm{BAO}+$ Pantheon+CC \\
\hline$\Omega_{c} h^{2}$ & $0.1192_{-0.0013-0.0027}^{+0.0014+0.0026}$ & $0.1194_{-0.0013-0.0025}^{+0.0013+0.0026}$ & $0.1195_{-0.0014-0.0027}^{+0.0013+0.0027}$ \\
\hline$\Omega_{b} h^{2}$ & $0.02223_{-0.00015-0.00030}^{+0.00015+0.00030}$ & $0.02223_{-0.00015-0.00028}^{+0.00015+0.00028}$ & $0.02222_{-0.00015-0.00029}^{+0.00015+0.00028}$ \\
\hline $100 \theta_{M C}$ & $1.04051_{-0.00034-0.00063}^{+0.00032+0.00064}$ & $1.04051_{-0.00033-0.00059}^{+0.00031+0.00061}$ & $1.04050_{-0.00033-0.00065}^{+0.00033+0.00062}$ \\
\hline$\tau$ & $0.079_{-0.018-0.035}^{+0.018+0.034}$ & $0.079_{-0.017-0.035}^{+0.017+0.033}$ & $0.081_{-0.018-0.035}^{+0.017+0.036}$ \\
\hline$n_{s}$ & $0.9744_{-0.0043-0.0088}^{+0.0043+0.0083}$ & $0.9740_{-0.0045-0.0082}^{+0.0042+0.0086}$ & $0.9740_{-0.0043-0.0086}^{+0.0045+0.0085}$ \\
\hline $\ln \left(10^{10} A_{s}\right)$ & $3.100_{-0.035-0.068}^{+0.035+0.067}$ & $3.100_{-0.033-0.067}^{+0.036+0.064}$ & $3.104_{-0.034-0.070}^{+0.034+0.069}$ \\
\hline$w_{0}$ & $-0.616_{-0.428-0.501}^{+0.326+0.560}$ & $-0.949_{-0.076-0.139}^{+0.074+0.149}$ & $-0.946_{-0.088-0.150}^{+0.072+0.155}$ \\
\hline$w_{a}$ & $-1.000_{-0.609-1.427}^{+1.039+1.262}$ & $-0.290_{-0.217-0.534}^{+0.277+0.460}$ & $-0.302_{-0.219-0.533}^{+0.310+0.500}$ \\
\hline$\Omega_{m 0}$ & $0.339_{-0.037-0.054}^{+0.031+0.058}$ & $0.305_{-0.008-0.015}^{+0.008+0.015}$ & $0.306_{-0.007-0.015}^{+0.008+0.015}$ \\
\hline$\sigma_{8}$ & $0.810_{-0.032-0.053}^{+0.030+0.055}$ & $0.839_{-0.016-0.031}^{+0.016+0.031}$ & $0.841_{-0.016-0.033}^{+0.016+0.033}$ \\
\hline$H_{0}$ & $64.98_{-3.35-5.47}^{+3.11+5.55}$ & $68.29_{-0.82-1.59}^{+0.81+1.65}$ & $68.24_{-0.78-1.50}^{+0.79+1.56}$ \\
\hline
\end{tabular}


Fig. $868 \%$ and $95 \% \mathrm{CL}$ contour plots for various combinations of the model parameters of the Ext2 of (8) have been shown for different observational combinations. The figure also contains the one dimensional marginalized posterior distributions for the parameters shown in the two dimensional contour plots

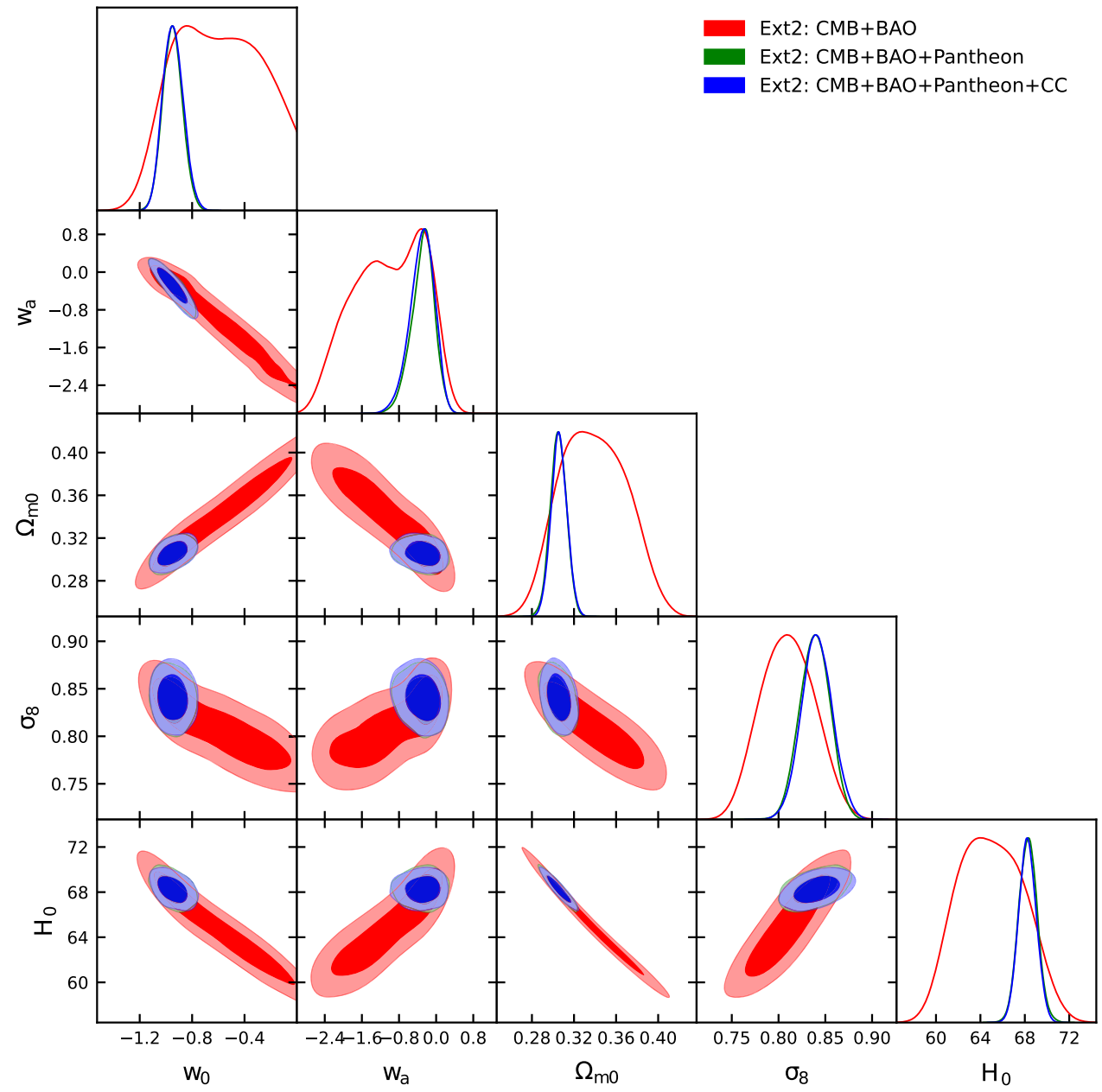

Table 5 Observational constraints on the dark energy parametrization, namely, the Ext3 of (9) using various observational datasets. We note that $H_{0}$ is in the units of $\mathrm{km} / \mathrm{Mpc} / \mathrm{sec}$ and $\Omega_{m 0}$ is the present value of $\Omega_{m}=\Omega_{b}+\Omega_{c}$

\begin{tabular}{|c|c|c|c|}
\hline Parameters & $\mathrm{CMB}+\mathrm{BAO}$ & $\mathrm{CMB}+\mathrm{BAO}+$ Pantheon & $\mathrm{CMB}+\mathrm{BAO}+\mathrm{Pantheon}+\mathrm{CC}$ \\
\hline$\Omega_{c} h^{2}$ & $0.1195_{-0.0013-0.0027}^{+0.0013+0.0027}$ & $0.1194_{-0.0013-0.0027}^{+0.0013+0.0026}$ & $0.1195_{-0.0013-0.0025}^{+0.0013+0.0027}$ \\
\hline$\Omega_{b} h^{2}$ & $0.02222_{-0.00014-0.00029}^{+0.00014+0.00029}$ & $0.02222_{-0.00014-0.00029}^{+0.00015+0.00030}$ & $0.02221_{-0.00015-0.00029}^{+0.00015+0.00029}$ \\
\hline $100 \theta_{M C}$ & $1.04049_{-0.00032-0.00065}^{+0.00031+0.00065}$ & $1.04050_{-0.00032-0.00065}^{+0.00033+0.00062}$ & $1.04047_{-0.00032-0.00063}^{+0.00032+0.00061}$ \\
\hline$\tau$ & $0.078_{-0.018-0.036}^{+0.018+0.035}$ & $0.080_{-0.017-0.035}^{+0.018+0.034}$ & $0.079_{-0.017-0.034}^{+0.018+0.033}$ \\
\hline$n_{s}$ & $0.9737_{-0.0048-0.0083}^{+0.0043+0.0089}$ & $0.9738_{-0.0043-0.0086}^{+0.0043+0.0086}$ & $0.9737_{-0.0043-0.0084}^{+0.0043+0.0084}$ \\
\hline $\ln \left(10^{10} A_{s}\right)$ & $3.099_{-0.036-0.069}^{+0.035+0.067}$ & $3.104_{-0.033-0.067}^{+0.034+0.066}$ & $3.101_{-0.034-0.067}^{+0.034+0.065}$ \\
\hline$w_{0}$ & $-0.582_{-0.501-0.564}^{+0.490+0.581}$ & $-0.946_{-0.080-0.137}^{+0.070+0.147}$ & $-0.936_{-0.071-0.141}^{+0.072+0.138}$ \\
\hline$w_{a}$ & $-1.077_{-1.010-1.450}^{+1.176+1.422}$ & $-0.295_{-0.215-0.508}^{+0.284+0.483}$ & $-0.335_{-0.222-0.472}^{+0.264+0.471}$ \\
\hline$\Omega_{m 0}$ & $0.343_{-0.045-0.059}^{+0.042+0.058}$ & $0.306_{-0.0075-0.015}^{+0.0075+0.015}$ & $0.306_{-0.0083-0.015}^{+0.0076+0.016}$ \\
\hline$\sigma_{8}$ & $0.808_{-0.036-0.052}^{+0.028+0.059}$ & $0.840_{-0.016-0.031}^{+0.016+0.031}$ & $0.841_{-0.017-0.034}^{+0.016+0.032}$ \\
\hline$H_{0}$ & $64.67_{-4.37-5.42}^{+3.73+6.18}$ & $68.26_{-0.82-1.58}^{+0.80+1.57}$ & $68.27_{-0.85-1.62}^{+0.83+1.73}$ \\
\hline
\end{tabular}

find any notable changes due to the extension of one more term in the DE parametrization. That means, the parametrization behaves similarly to the previous two parametrizations.

Finally, we focus on the last parametrization of this series, namely Ext3 shown in Eq. (9). We have summarized the results in Table 5 and Fig. 9, using the same combinations of the cosmological datasets that have been used for the previous parametrizations. It is interesting to note that even if we successively increase the terms in the Taylor expansion of the generalized parametrization (6), but that does not attribute to any change in the constraints on the key parameters as well as on the derived parameters, for instance the Hubble constant. 
Fig. $968 \%$ and $95 \% \mathrm{CL}$ contour plots for various combinations of the model parameters of the Ext3 of (9) have been shown for different observational combinations. The figure also contains the one dimensional marginalized posterior distributions for the parameters shown in the two dimensional contour plots

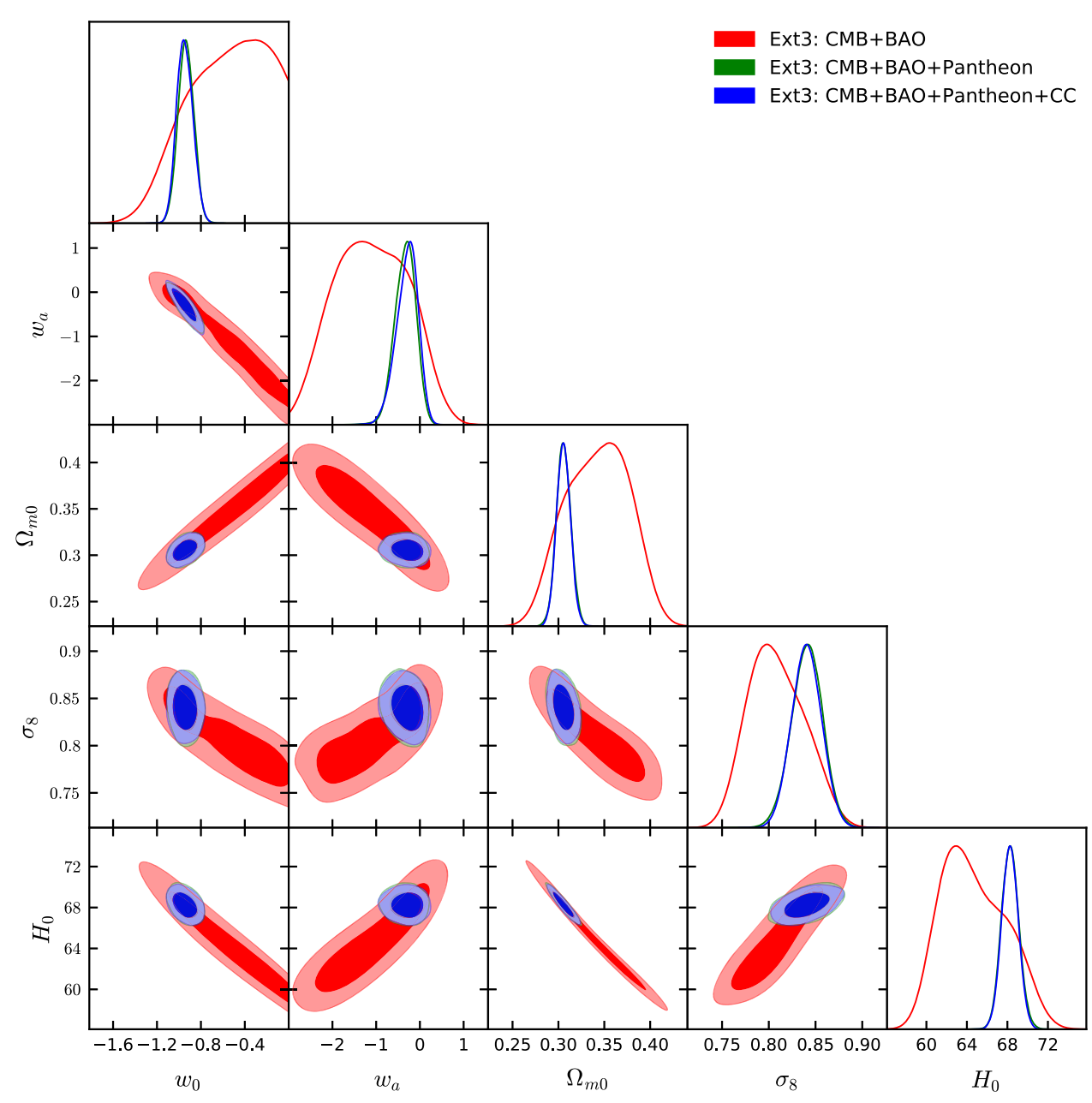

For a better understanding on the key parameters $\left(w_{0}, w_{a}\right)$ obtained from various combined datasets, in Table 6, we have presented their $68 \%$ CL constraints and in Fig. 10, we have shown their two dimensional contour plots. The Table 6 and Fig. 10 clearly emphasize that at the background level, none of the extensions can be distinguished from the general parametrization. However, as we will show below that, at the level of perturbations, the inclusion of higher order terms certainly exhibits some changes.

Thus, we investigate how the present dark energy paramebreaktrization, namely, the new dark energy parametrization in Eq. (6), and its extensions in Eqs. (7), (8) and (9) affect various observables, such as the temperature anisotropy in the cosmic microwave background spectra as well as the matter power spectra. Such an investigation is important since this enables one to understand how the higher order extensions of the original dark energy parametrization (6) affect the structure formation of the universe. Thus, in Fig. 11 we show the temperature anisotropy in the CMB spectra and the residual plots for different dark energy parametrizations for various values of $w_{a}$ parameter with a fixed value of $w_{0}=-0.95$. We have actually fixed $w_{0}=-0.95$ since from the obser- vational analyses of the models presented in various tables of this article, $w_{0}$ assumes values close to -0.95 . For completeness, we have considered both the possibilities namely $w_{a}>0$ and $w_{a}<0$. The plots in the first row of Fig. 11 depict the temperature anisotropy in the CMB spectra for $w_{a}>0$ and the plots in the second row of Fig. 11 describe the corresponding residual plots. Let us note that the plots from left to right in both the first and second rows of Fig. 11 respectively stand for $w_{a}=0.1,0.2$, and 0.3 . In a similar fashion, the plots in the third row of Fig. 11 stands for the CMB spectra assuming $w_{a}<0$ and the plots in the last row of Fig. 11 represent the corresponding residual plots. And the plots from left to right in both the third and last rows of Fig. 11 respectively stand for $w_{a}=-0.1,-0.2$ and -0.3 . From the first row of Fig. 11 one can clearly notice that the DE parametrizations cannot be distinguished from one another, even if we increase the magnitude of $w_{a}$, however, when we look at the corresponding residual plots shown in the second row of Fig. 11, we realize the differences. It is clear that Ext3 is more close to the original parametrization (6) compared to Ext1 and Ext2. The same conclusion can be drawn from the 

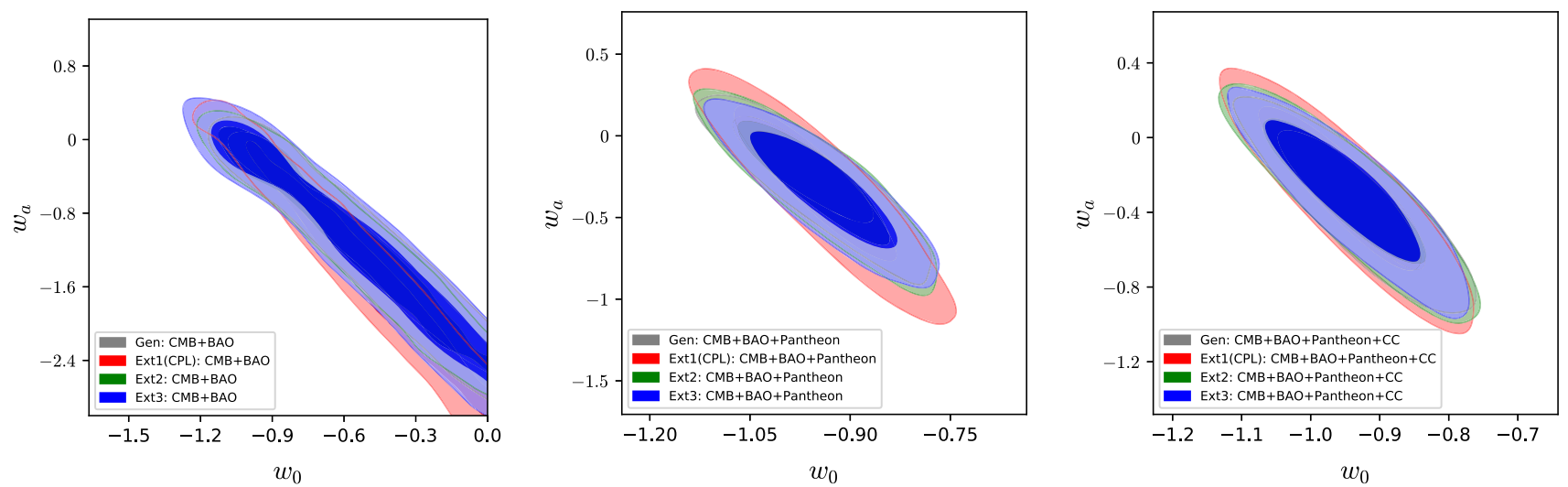

Fig. 10 We show the $\left(w_{0}, w_{a}\right)$ plane for the present dynamical dark energy parametrizations using different observational datasets. The left graph for the dataset $\mathrm{CMB}+\mathrm{BAO}$, the middle graph for the dataset $\mathrm{CMB}+\mathrm{BAO}+$ Pantheon and the right graph stands for the observational dataset $\mathrm{CMB}+\mathrm{BAO}+$ Pantheon+CC

Table 6 For a clear understanding and comparison of the dark energy key parameters $\left(w_{0}, w_{a}\right)$ extracted from all the parametrizations, we show their estimations at $68 \% \mathrm{CL}$. Here, $\mathrm{CB}=\mathrm{CMB}+\mathrm{BAO}, \mathrm{CBP}=\mathrm{CMB}+\mathrm{BAO}+\mathrm{Pantheon}, \mathrm{CBPC}=\mathrm{CMB}+\mathrm{BAO}+\mathrm{Pantheon}+\mathrm{CC}$

\begin{tabular}{llllll}
\hline Datasets Parameters Gen & Ext1 $(\mathrm{CPL})$ & Ext2 & Ext3 \\
\hline $\mathrm{CB}$ & $\left(w_{0}, w_{a}\right)$ & $\left(-0.537_{-0.441}^{+0.442},-1.154_{-0.807}^{+0.894}\right)$ & $\left(-0.524_{-0.236}^{+0.374},-1.403_{-1.021}^{+0.731}\right)$ & $\left(-0.616_{-0.428}^{+0.326},-1.000_{-0.609}^{+1.039}\right)$ & $\left(-0.582_{-0.501}^{+0.490},-1.077_{-1.010}^{+1.176}\right)$ \\
$\mathrm{CBP}$ & $\left(w_{0}, w_{a}\right)$ & $\left(-0.963_{-0.082}^{+0.060},-0.231_{-0.143}^{+0.291}\right)$ & $\left(-0.947_{-0.088}^{+0.076},-0.308_{-0.273}^{+0.367}\right)$ & $\left(-0.949_{-0.076}^{+0.074},-0.290_{-0.217}^{+0.277}\right)$ & $\left(-0.946_{-0.080}^{+0.070},-0.295_{-0.215}^{+0.284}\right)$ \\
$\mathrm{CMPC}$ & $\left(w_{0}, w_{a}\right)$ & $\left(-0.933_{-0.070}^{+0.071},-0.337_{-0.204}^{+0.288}\right)$ & $\left(-0.950_{-0.084}^{+0.075},-0.291_{-0.256}^{+0.338}\right)$ & $\left(-0.946_{-0.088}^{+0.072},-0.302_{-0.219}^{+0.310}\right)$ & $\left(-0.936_{-0.071}^{+0.072},-0.335_{-0.222}^{+0.264}\right)$ \\
\hline
\end{tabular}

last row of Fig. 11. So, effectively, independently of the sign of $w_{a}$, the conclusion remains same.

Following a similar graphical strategy applied to matter power spectra plots as shown in Fig. 12, we arrive at the same conclusion that the models are only distinguished from one another if we look at the residual plots, that means the plots summarized in the second and last rows of Fig. 12.

\section{Bayesian evidence}

A general and natural question that we will be looking for in this section is that, how the models are efficient compared to the standard $\Lambda$ CDM cosmology. Thus, we need a statistical comparison between all four dynamical DE parametrizations where the base model will be fixed as $\Lambda \mathrm{CDM}$. This statistical comparison comes through the Bayesian evidence. Here we apply publicly available code MCEvidence $[61,62]^{1}$ to compute the evidences of the models. The use of MCEvidence is very easy since the code only needs the MCMC chains used to extract the free parameters of the DE parametrizations.

While dealing with Bayesian analysis we need the posterior probability of the model parameters (denoted by $\theta$ ), given a specific observational data $(x)$ with any prior information for a model $(M)$. Following Bayes theorem, one can

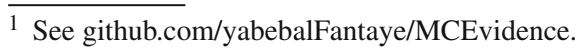

write,

$p(\theta \mid x, M)=\frac{p(x \mid \theta, M) \pi(\theta \mid M)}{p(x \mid M)}$,

where $p(x \mid \theta, M)$ is the likelihood as a function of $\theta$ and $\pi(\theta \mid M)$ refers to the prior information. Here, the quantity $p(x \mid M)$ appearing in the denominator of (24) is the Bayesian evidence that we actually need for the model comparison. Now, for two cosmological models $M_{i}, M_{j}$ where $M_{j}$ is acting as the reference model, ${ }^{2}$ the posterior probability is,

$\frac{p\left(M_{i} \mid x\right)}{p\left(M_{j} \mid x\right)}=\frac{\pi\left(M_{i}\right)}{\pi\left(M_{j}\right)} \frac{p\left(x \mid M_{i}\right)}{p\left(x \mid M_{j}\right)}=\frac{\pi\left(M_{i}\right)}{\pi\left(M_{j}\right)} B_{i j}$,

in which $B_{i j}=\frac{p\left(x \mid M_{i}\right)}{p\left(x \mid M_{j}\right)}$, is the Bayes factor of the model $M_{i}$ relative to $M_{j}$. And based on the values of $B_{i j}$ (alternatively, $\ln B_{i j}$ ) we quantify the observational support of the underlying model $M_{i}$ relative to $M_{j}$. The quantification is done through the widely accepted Jeffreys scales [63] (see Table 7). We also note that the negative values of $\ln B_{i j}$ indicate that the reference model $\left(M_{j}\right)$ is preferred over the underlying model $\left(M_{i}\right)$.

\footnotetext{
2 The reference model should be the most well motivated cosmological model that must be highly sound to the observational data; and without any doubt, $\Lambda \mathrm{CDM}$ is the best choice for such a model comparison.
} 

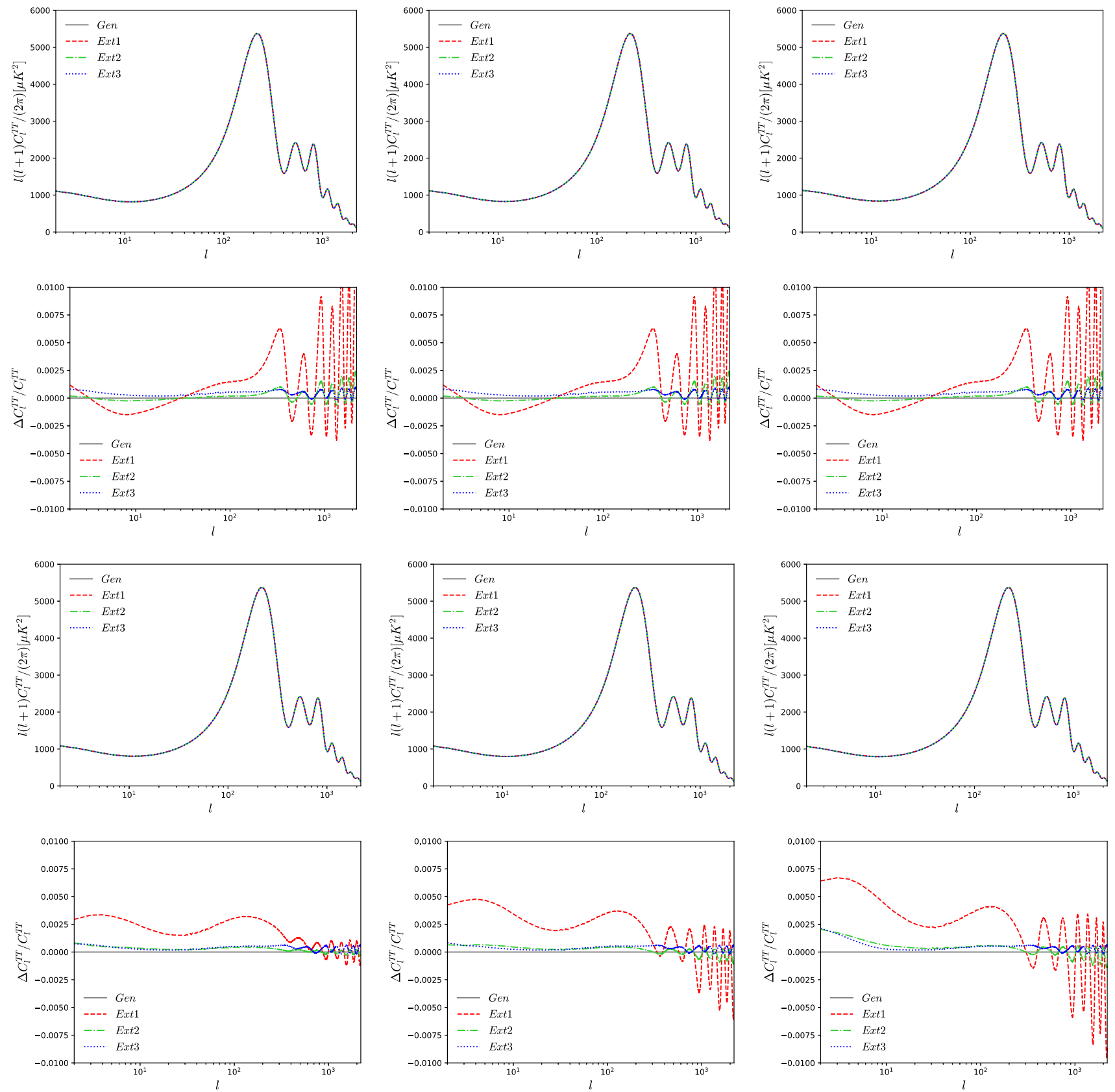

Fig. 11 We show the cosmic microwave background spectra and the corresponding residual plots for the present dynamical dark energy parameterizations using various values of the $w_{a}$ parameter with a fixed $w_{0}=-0.95$. The plots in the first row present the cosmic microwave background spectra and the plots in the second row present the corresponding residual plots. The plots from left to right in the first and second

panels of this figure respectively stand for $w_{a}=0.1,0.2$, and 0.3. Similarly, the plots in the third row (showing the cosmic microwave background spectra) and last row (residual plots of the third row) stand for $w_{a}<0$ in which the plots from left to right for both the above rows (third and last rows) of this figure respectively stand for $w_{a}=-0.1,-0.2$, and -0.3

In Table 8 we have shown the values of $\ln B_{i j}$ computed for all DE parametrizations considering all the datasets. We find that the values of $\ln B_{i j}$ are all negative indicating that $\Lambda \mathrm{CDM}$ is always preferred and this is true for all the observational datasets.

\section{Concluding remarks}

The dark energy, a hypothetical fluid in Einstein gravity is the main concern of this work. This dark energy, as examined by many investigators since the year 1998, could be anything obeying only one condition that the pressure of the 

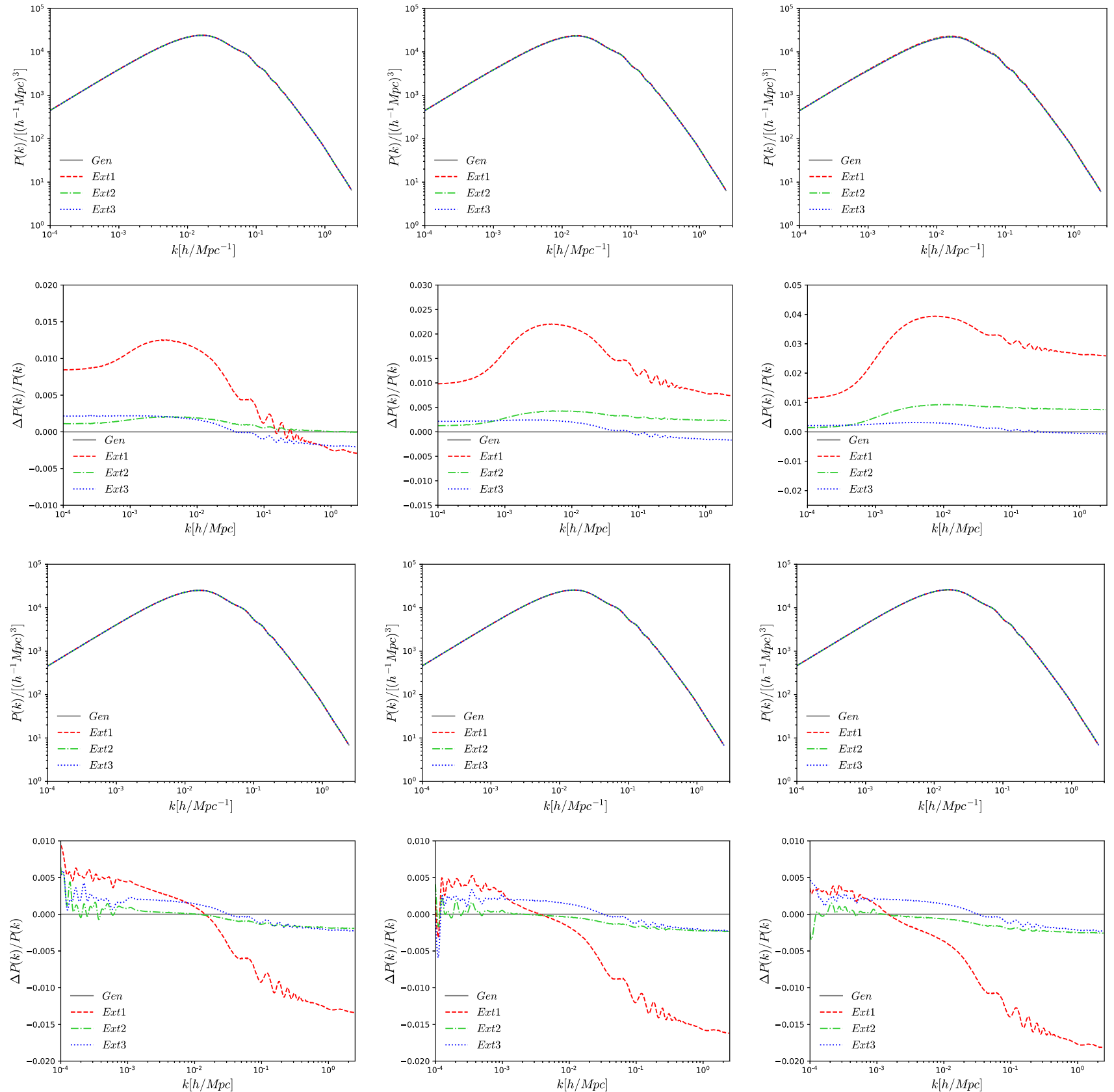

Fig. 12 We show the matter power spectra and the corresponding residual plots for the present dynamical dark energy parameterizations using various values of the $w_{a}$ parameter with a fixed $w_{0}=-0.95$. The plots in the first row present the matter power spectra and the plots in the second row present the corresponding residual plots. The plots from left to right in the first and second panels of this figure respectively stand for $w_{a}=0.1,0.2$, and 0.3. Similarly, the plots in the third row (showing the matter power spectra) and last row (residual plots of the third row) stand for $w_{a}<0$ in which the plots from left to right for both the above rows (third and last rows) of this figure respectively stand for $w_{a}=-0.1,-0.2$, and -0.3 fluid should be negative. Thereafter, a cluster of dark energy models have been introduced and confronted with the observational data, see [1] to get an overview of the models.

Among them an interesting construction of the dark energy models comes through the equation of state of dark energy, $w_{x}=p_{x} / \rho_{x}$ which in principle is the function of the underlying cosmological time parameter, usually the function of the redshift. Technically, there is no such restriction to pick up any specific functional form for $w_{x}$, however, the viability of the model is only tested through the observational data and its effects on the large scale structure of the universe indeed. According to the investigations per- 
Table 7 Revised Jeffreys scale quantifying the observational viability of any cosmological model $M_{i}$ compared to some reference model $M_{j}$ [63]

\begin{tabular}{ll}
\hline $\ln B_{i j}$ & Evidence for model $M_{i}$ \\
\hline $0 \leq \ln B_{i j}<1$ & Weak \\
$1 \leq \ln B_{i j}<3$ & Definite/positive \\
$3 \leq \ln B_{i j}<5$ & Strong \\
$\ln B_{i j} \geq 5$ & Very strong \\
\hline
\end{tabular}

formed in the last couple of years, the Chevallier-PolarskiLinder parametrization is a feasible and well functioning dark energy parametrization with the observational data. The present work is motivated in the same direction whilst we have investigated something different as follows.

We have introduced a new dark energy parametrization (6) having a novel feature. The model recovers the well known CPL parametrization in its first order Taylor series expansion around $z=0$. Thus, the model actually presents a generalized version of the CPL parametrization. Since the model is a nonlinear generalized version of the CPL model, thus, a natural inquiry one may ask for is, how its higher order corrections are important for the expansion history of the universe, and moreover, how the higher order corrections could affect the evolution of the universe at the level of background and perturbations. In order to investigate these issues, we have considered the generalized model (6) together with its first, second and third order Taylor approximations around the present cosmic epoch $z=0$, given in Eqs. (7), (8) and (9). Since the original model (6) contains only two free parameters $w_{0}$ (current value of the dark energy equation of state) and $w_{a}$ (parameter quantifying the dynamical nature of the $\mathrm{DE}$ ), thus its extensions contain the same free parameters. We then constrain all the models using a class of astronom- ical data, such as CMB, BAO, Pantheon from SNIa and the Hubble parameter measurements (summarized in Sect. 4).

The observational constraints are summarized in Table 2 (for Eq. (6)), Table 3 (for Eq. (7)), Table 4 (for Eq. (8)), Table 5 (for Eq. (9)) and the graphical variations of the model parameters are also shown in Figs. 6, 7, 8 and 9, respectively for the general, Ext1, Ext2, and Ext3 parametrizations. From the analyses, it is clear that the cosmological parameters assume similar constraints and according to the employed observational data applied to the present models, the dark energy equation of state at present, $w_{0}$, is consistent to $w_{0}=-1$ scenario. In addition, we find that, for $\mathrm{CMB}+\mathrm{BAO}$ data, $H_{0}$ for all parametrizations, assumes very lower values, if we disregard its error bars, however, for $\mathrm{CMB}+\mathrm{BAO}+$ Pantheon and $\mathrm{CMB}+\mathrm{BAO}+\mathrm{Pantheon}+\mathrm{CC}, H_{0}$ increases with slightly higher error bars compared to the $\Lambda$ CDM based Planck's estimation [59], and as a result the tension on $H_{0}$ is slightly reduced. However, at the level of background, the models cannot be distinguished from one another while from the investigations at perturbations stage, one can distinguish between the models, see the residual plots in Figs. 11 and 12.

We also performed the Bayesian evidence analysis using the MCEvidence and compared the models with respect to the standard $\triangle \mathrm{CDM}$ reference scenario. Our analysis reveals that $\Lambda \mathrm{CDM}$ is favored over all the dynamical $\mathrm{DE}$ parametrizations. This is an expected result because the parameters space of the leading cosmic scenarios driven by the present dynamical DE parametrizations are of eight dimensional while $\Lambda \mathrm{CDM}$ has only six parameters.

Last but not least, we would like to comment that the model (6), so far we are aware of the literature, is a new one in the field of dark energy which naturally recovers CPL parametrization in its first order approximation and sounds good with the Bayesian evidence. Therefore, a number of investigations can be performed in various contexts of cur-
Table 8 The values of $\ln B_{i j}$ computed for the present dark energy parametrizations $\left(M_{i}\right)$ where the reference scenario is the $\Lambda$ CDM model $\left(M_{j}\right)$. We note that the negative value of $\ln B_{i j}$ means that $\Lambda \mathrm{CDM}$ is preferred

\begin{tabular}{llll}
\hline Dataset & Model & $\ln B_{i j}$ & Evidence for $\Lambda$ CDM \\
\hline CMB+BAO & Gen & -1.4 & Definite \\
CMB+BAO+Pantheon & Gen & -3.4 & Strong \\
CMB+BAO+Pantheon+CC & Gen & -3.6 & Strong \\
CMB+BAO & Ext1 & -1.1 & Definite \\
CMB+BAO+Pantheon & Ext1 & -3.3 & Strong \\
CMB+BAO+Pantheon+CC & Ext1 & -3.4 & Strong \\
CMB+BAO & Ext2 & -1.7 & Definite \\
CMB+BAO+Pantheon & Ext2 & -3.2 & Strong \\
CMB+BAO+Pantheon+CC & Ext2 & -3.3 & Strong \\
CMB+BAO & Ext3 & -1.6 & Definite \\
CMB+BAO+Pantheon & Ext3 & -3.9 & Strong \\
CMB+BAO+Pantheon+CC & Ext3 & -3.5 & Strong \\
\hline
\end{tabular}


rent interests. A quite straightforward and appealing investigation would be to measure the mass bounds of neutrinos in such a generalized framework. Moreover, it will be further interesting to consider a number of upcoming cosmological surveys, such as, Simons Observatory Collaboration (SOC) [64], Cosmic Microwave Background Stage-4 (CMBS4) [65], EUCLID Collaboration [66, 67], Dark Energy Spectroscopic Instrument (DESI) [68], Large Synoptic Survey Telescope (LSST) [69-71], in order to forecast the present dark energy parametrizations. The inclusion of gravitational waves data from various sources, such as, Laser Interferometer Space Antenna (LISA) [72], Deci-hertz Interferometer Gravitational wave Observatory (DECIGO) [73,74], TianQin [75], is also an appealing direction of research in this direction.

Acknowledgements The authors thank the referee for some useful and important comments that helped to improve the quality of the manuscript. SP acknowledges the financial support through the Faculty Research and Professional Development Fund (FRPDF) Scheme of Presidency University, Kolkata, India. WY was supported by the financial support from the National Natural Science Foundation of China under Grant nos. 11705079 and 11647153.

Data Availability Statement This manuscript has no associated data or the data will not be deposited. [Authors' comment: There are not new data to deposit. The data we used are given in the references as presented in the text.]

Open Access This article is licensed under a Creative Commons Attribution 4.0 International License, which permits use, sharing, adaptation, distribution and reproduction in any medium or format, as long as you give appropriate credit to the original author(s) and the source, provide a link to the Creative Commons licence, and indicate if changes were made. The images or other third party material in this article are included in the article's Creative Commons licence, unless indicated otherwise in a credit line to the material. If material is not included in the article's Creative Commons licence and your intended use is not permitted by statutory regulation or exceeds the permitted use, you will need to obtain permission directly from the copyright holder. To view a copy of this licence, visit http://creativecomm ons.org/licenses/by/4.0/.

Funded by SCOAP ${ }^{3}$.

\section{References}

1. E.J. Copeland, M. Sami, S. Tsujikawa, Dynamics of dark energy. Int. J. Mod. Phys. D 15, 1753 (2006)

2. M. Chevallier, D. Polarski, Accelerating universes with scaling dark matter. Int. J. Mod. Phys. D 10, 213 (2001)

3. E.V. Linder, Exploring the expansion history of the universe. Phys. Rev. Lett. 90, 091301 (2003)

4. A.R. Cooray, D. Huterer, Gravitational lensing as a probe of quintessence. Astrophys. J. 513, L95 (1999)

5. G. Efstathiou, Constraining the equation of state of the universe from distant type Ia supernovae and cosmic microwave background anisotropies. Mon. Not. R. Astron. Soc. 310, 842 (1999)

6. P. Astier, Can luminosity distance measurements probe the equation of state of dark energy. Phys. Lett. B 500, 8 (2001)
7. J. Weller, A. Albrecht, Future supernovae observations as a probe of dark energy. Phys. Rev. D 65, 103512 (2002)

8. C. Wetterich, Phenomenological parameterization of quintessence. Phys. Lett. B 594, 17 (2004)

9. S. Hannestad, E. Mortsell, Cosmological constraints on the dark energy equation of state and its evolution. JCAP 0409, 001 (2004)

10. H.K. Jassal, J.S. Bagla, T. Padmanabhan, Observational constraints on low redshift evolution of dark energy: how consistent are different observations? Phys. Rev. D 72, 103503 (2005)

11. Yg Gong, Y.Z. Zhang, Probing the curvature and dark energy. Phys. Rev. D 72, 043518 (2005)

12. B. Feng, M. Li, Y.S. Piao, X. Zhang, Oscillating quintom and the recurrent universe. Phys. Lett. B 634, 101 (2006)

13. S. Nojiri, S.D. Odintsov, The oscillating dark energy: future singularity and coincidence problem. Phys. Lett. B 637, 139 (2006)

14. G.B. Zhao, J.Q. Xia, H. Li, C. Tao, J.M. Virey, Z.H. Zhu, X. Zhang, Probing for dynamics of dark energy and curvature of universe with latest cosmological observations. Phys. Lett. B 648, 8 (2007)

15. A. Kurek, O. Hrycyna, M. Szydlowski, Constraints on oscillating dark energy models. Phys. Lett. B 659, 14 (2008)

16. E.M. Barboza Jr., J.S. Alcaniz, A parametric model for dark energy. Phys. Lett. B 666, 415 (2008)

17. E.N. Saridakis, Theoretical limits on the equation-of-state parameter of phantom cosmology. Phys. Lett. B 676, 7 (2009)

18. R. Lazkoz, V. Salzano, I. Sendra, Oscillations in the dark energy EoS: new MCMC lessons. Phys. Lett. B 694, 198 (2010)

19. J.Z. Ma, X. Zhang, Probing the dynamics of dark energy with novel parametrizations. Phys. Lett. B 699, 233 (2011)

20. H. Li, X. Zhang, Probing the dynamics of dark energy with divergence-free parametrizations: a global fit study. Phys. Lett. B 703, 119 (2011)

21. L. Feng, T. Lu, A new equation of state for dark energy model. JCAP 1111, 034 (2011)

22. I. Sendra, R. Lazkoz, SN and BAO constraints on (new) polynomial dark energy parametrizations: current results and forecasts. Mon. Not. R. Astron. Soc. 422, 776 (2012)

23. C.J. Feng, X.Y. Shen, P. Li, X.Z. Li, A new class of parametrization for dark energy without divergence. JCAP 1209, 023 (2012)

24. E. Di Valentino, A. Melchiorri, J. Silk, Reconciling Planck with the local value of $H_{0}$ in extended parameter space. Phys. Lett. B 761, 242 (2016)

25. G.B. Zhao et al., Dynamical dark energy in light of the latest observations. Nat. Astron. 1, 627 (2017)

26. E. Di Valentino, A. Melchiorri, E.V. Linder, J. Silk, Constraining dark energy dynamics in extended parameter space. Phys. Rev. D 96, 023523 (2017)

27. E. Di Valentino, Crack in the cosmological paradigm. Nat. Astron. 1, 569 (2017)

28. W. Yang, R.C. Nunes, S. Pan, D.F. Mota, Effects of neutrino mass hierarchies on dynamical dark energy models. Phys. Rev. D 95 , 103522 (2017)

29. M. Rezaei, M. Malekjani, S. Basilakos, A. Mehrabi, D.F. Mota, Constraints to dark energy using PADE parameterizations. Astrophys. J. 843(1), 65 (2017)

30. R.J.F. Marcondes, S. Pan, Cosmic chronometers constraints on some fast-varying dark energy equations of state. arXiv:1711.06157 [astro-ph.CO]

31. W. Yang, S. Pan, A. Paliathanasis, Latest astronomical constraints on some nonlinear parametric dark energy models. Mon. Not. R. Astron. Soc. 475, 2605 (2018)

32. M. Jaber, A. de la Macorra, Probing a steep EoS for dark energy with latest observations. Astropart. Phys. 97, 130 (2018)

33. S. Pan, E.N. Saridakis, W. Yang, Observational constraints on oscillating dark-energy parametrizations. Phys. Rev. D 98(6), 063510 (2018) 
34. S. Vagnozzi, S. Dhawan, M. Gerbino, K. Freese, A. Goobar, O. Mena, Constraints on the sum of the neutrino masses in dynamical dark energy models with $w(z) \geq-1$ are tighter than those obtained in $\Lambda$ CDM. Phys. Rev. D 98(8), 083501 (2018)

35. X.D. Li, Cosmological constraints from the redshift dependence of the Alcock-Paczynski effect: dynamical dark energy. Astrophys. J. 856(2), 88 (2018)

36. G. Panotopoulos, Á. Rincón, Growth index and statefinder diagnostic of oscillating dark energy. Phys. Rev. D 97(10), 103509 (2018)

37. L.G. Jaime, M. Jaber, C. Escamilla-Rivera, New parametrized equation of state for dark energy surveys. Phys. Rev. D 98(8), 083530 (2018)

38. W. Yang, S. Pan, E. Di Valentino, E.N. Saridakis, S. Chakraborty, Observational constraints on one-parameter dynamical darkenergy parametrizations and the $H_{0}$ tension. arXiv:1810.05141 [astro-ph.CO]

39. W. Yang, S. Pan, E. Di Valentino, E.N. Saridakis, Observational constraints on dynamical dark energy with pivoting redshift. arXiv:1811.06932 [astro-ph.CO]

40. F. Pace, C. Schimd, D.F. Mota, A. Del Popolo, Halo collapse: virialization by shear and rotation in dynamical dark-energy models. arXiv:1811.12105 [astro-ph.CO]

41. M. Du, W. Yang, L. Xu, S. Pan, D.F. Mota, Future constraints on dynamical dark-energy using gravitational-wave standard sirens. arXiv:1812.01440 [astro-ph.CO]

42. D. Tamayo, J.A. Vazquez, Fourier series expansion of the dark energy equation of state. arXiv:1901.08679 [astro-ph.CO]

43. V.F. Mukhanov, H.A. Feldman, R.H. Brandenberger, Theory of cosmological perturbations. Phys. Rep. 215, 203 (1992)

44. C.P. Ma, E. Bertschinger, Cosmological perturbation theory in the synchronous and conformal Newtonian gauges. Astrophys. J. 455, 7 (1995)

45. K.A. Malik, D. Wands, Cosmological perturbations. Phys. Rep. 475, 1 (2009)

46. N. Dimakis, A. Karagiorgos, A. Zampeli, A. Paliathanasis, T. Christodoulakis, P.A. Terzis, General analytic solutions of scalar field cosmology with arbitrary potential. Phys. Rev. D 93, 123518 (2016)

47. R. Adam et al. [Planck Collaboration], Planck 2015 results. I. Overview of products and scientific results. Astron. Astrophys. 594, A1 (2016)

48. N. Aghanim et al. [Planck Collaboration], Planck 2015 results. XI. CMB power spectra, likelihoods, and robustness of parameters. Astron. Astrophys. 594, A11 (2016)

49. M. Betoule et al., [SDSS Collaboration], Improved cosmological constraints from a joint analysis of the SDSS-II and SNLS supernova samples. Astron. Astrophys. 568, A22 (2014)

50. F. Beutler et al., The $6 \mathrm{dF}$ Galaxy Survey: baryon acoustic oscillations and the local hubble constant. Mon. Not. R. Astron. Soc. 416 3017 (2011)

51. A.J. Ross, L. Samushia, C. Howlett, W.J. Percival, A. Burden, M. Manera, The clustering of the SDSS DR7 main Galaxy sample â€I. A 4 per cent distance measure at $z=0.15$. Mon. Not. R. Astron. Soc. 449(1), 835 (2015)

52. H. Gil-Marín et al., The clustering of galaxies in the SDSS-III Baryon Oscillation Spectroscopic Survey: BAO measurement from the LOS-dependent power spectrum of DR12 BOSS galaxies. Mon. Not. R. Astron. Soc. 460(4), 4210 (2016)

53. D.M. Scolnic et al., The complete light-curve sample of spectroscopically confirmed SNe Ia from Pan-STARRS1 and cosmological constraints from the combined pantheon sample. Astrophys. J. 859(2), 101 (2018)
54. M. Moresco et al., A $6 \%$ measurement of the Hubble parameter at $z \sim 0.45$ : direct evidence of the epoch of cosmic re-acceleration. JCAP 1605(05), 014 (2016)

55. A. Lewis, S. Bridle, Cosmological parameters from CMB and other data: a Monte Carlo approach. Phys. Rev. D 66, 103511 (2002)

56. A. Lewis, A. Challinor, A. Lasenby, Efficient computation of CMB anisotropies in closed FRW models. Astrophys. J. 538, 473 (2000)

57. A. Gelman, D. Rubin, Inference from iterative simulation using multiple sequences. Stat. Sci. 7, 457 (1992)

58. N. Aghanim [Planck Collaboration], Planck, et al., Results (VI, Cosmological parameters, 2018). arXiv:1807.06209 [astro-ph.CO]

59. P.A.R. Ade et al. [Planck Collaboration], Planck 2015 results. XIII. Cosmological parameters. Astron. Astrophys. 594, A13 (2016). arXiv:1502.01589 [astro-ph.CO]

60. A.G. Riess et al., A $2.4 \%$ determination of the local value of the Hubble constant. Astrophys. J. 826(1), 56 (2016). arXiv:1604.01424 [astro-ph.CO]

61. A. Heavens, Y. Fantaye, A. Mootoovaloo, H. Eggers, Z. Hosenie, S. Kroon, E. Sellentin, Marginal likelihoods from Monte Carlo Markov chains. arXiv:1704.03472 [stat.CO]

62. A. Heavens, Y. Fantaye, E. Sellentin, H. Eggers, Z. Hosenie, S. Kroon, A. Mootoovaloo, No evidence for extensions to the standard cosmological model. Phys. Rev. Lett. 119(10), 101301 (2017)

63. R.E. Kass, A.E. Raftery, Bayes factors. J. Am. Stat. Assoc. 90(430), 773 (1995)

64. J. Aguirre et al., Simons Observatory Collaboration, The Simons Observatory: science goals and forecasts. JCAP 1902, 056 (2019)

65. M.H. Abitbol et al. [CMB-S4 Collaboration], CMB-S4 Technology Book, 1st edn. arXiv:1706.02464 [astro-ph.IM]

66. R. Laureijs et al. [EUCLID Collaboration], Euclid Definition Study Report. arXiv:1110.3193 [astro-ph.CO]

67. R. Scaramella et al., [Euclid Collaboration], Euclid space mission: a cosmological challenge for the next 15 years. IAU Symp. 10, 375 (2014)

68. A. Aghamousa et al. [DESI Collaboration], The DESI experiment part I: science,targeting, and survey design. arXiv:1611.00036 [astro-ph.IM]

69. J.A. Newman et al. [LSST Dark Energy Science Collaboration], Deep multi-object spectroscopy to enhance dark energy science from LSST. arXiv:1903.09325 [astro-ph.CO]

70. R.A. Hložek et al. [LSST Dark Energy Science Collaboration], Single-object imaging and spectroscopy to enhance dark energy science from LSST. arXiv:1903.09324 [astro-ph.CO]

71. R. Mandelbaum et al. [LSST Dark Energy Science Collaboration], Wide-field multi-object spectroscopy to enhance dark energy science from LSST. arXiv:1903.09323 [astro-ph.CO]

72. H. Audley et al. [LISA Collaboration], Laser interferometer space antenna. arXiv:1702.00786 [astro-ph.IM]

73. S. Kawamura et al., The Japanese space gravitational wave antenna: DECIGO. Class. Quantum Gravity 28, 094011 (2011)

74. S. Sato et al., The status of DECIGO. J. Phys. Conf. Ser. 840(1), 012010 (2017)

75. J. Luo et al. [TianQin Collaboration], TianQin: a space-borne gravitational wave detector. Class. Quantum Gravity 33(3), 035010 (2016) 